\title{
PERISTALTIC FLOW AND HEAT TRANSFER OF A CONDUCTING PHAN-THIEN-TANNER FLUID IN AN ASYMMETRIC CHANNEL - APPLICATION TO CHYME MOVEMENT IN SMALL INTESTINE
}

\author{
K. VAJRAVELU* \\ Department of Mathematics \\ Department of Mechanical, Materials and Aerospace Engineering \\ University of Central Florida \\ Orlando, Florida 32816-1364, USA \\ E-mail: kuppalapalle.vajravelu@ucf.edu \\ S. SREENADH and S. DHANANJAYA \\ Department of Mathematics \\ Sri Venkateswara University \\ Tirupati 517 502, INDIA \\ E-mail: drsreenadh@yahoo.co.in \\ P. LAKSHMINARAYANA \\ Department of Mathematics \\ SreeVidyanikethan Engineering College \\ Tirupati 517 102, INDIA \\ E-mail: laxminarayana.pallava@gmail.com
}

\begin{abstract}
In this paper, the influence of heat transfer on the peristaltic flow of a conducting Phan-Thien-Tanner fluid in an asymmetric channel with porous medium is studied. The coupled nonlinear governing differential equations are solved by a perturbation technique. The expressions for the temperature field, the stream function, the axial velocity, and the pressure gradient are obtained. The effects of the various physical parameters such as the magnetic parameter $M$, the permeability parameter $\sigma$, the Brinkman number $\mathrm{Br}$ and the Weissenberg number $\mathrm{We}$ on the pumping phenomenon are analyzed through graphs and the results are discussed in detail. It is observed that the velocity and the pressure are decreased with increasing the magnetic parameter $M$ whereas the effect of the parameter $M$ on the temperature field is quite the opposite.
\end{abstract}

Key words: peristaltic flow, Phan-Thien-Tanner fluid, asymmetric channel, porous medium, trapping phenomena.

Mathematics Subject Classification 2010: 76A05, 92B05.

\section{Introduction}

Peristaltic transport is a natural mechanism of pumping induced by a progressive wave of area contraction or expansion along the length of the boundary of a fluid-filled distensible tube. This principle occurs in many biological and biomedical systems such as the transport of urine from kidney to the bladder, transport of spermatozoa in the ductus efferentes of the male reproductive tract, transport of lymph in the lymphatic vessels, the movement of chyme in the gastrointestinal tract movement of the

\footnotetext{
* To whom correspondence should be addressed
} 
ovum in the female fallopian tube, vasomotion of small blood vessels such as arterioles, venues and capillaries, and so on. Also this mechanism finds applications in blood pumps, heart-lung machines, dialysis machines, roller and finger pumps, and also noxious fluid transport in nuclear industries. The peristaltic flow of non-Newtonian fluids has gained considerable interest during recent years because of its applications in industry and biology. Some of the studies on the peristaltic flow of non-Newtonian fluids can be seen in references (Misra and Pandey [1], Usha and Rao [2]; Mishra and Rao [3]; Hayat et al. [4]; Noreen and Nadeem [5]; Vajravelu et al. [6]).

Flows through a porous medium occur in filtration of fluids and seepage of water under a dam. Consideration of porosity is very necessary to properly explain the fluid dynamical process that occurs in different parts of a living body, such as vascular systems, lungs, kidneys, and blood vessels. The peristaltic transport and heat transfer related to the flow of blood and some other fluids through porous media has been discussed by different researchers. Vajravelu et al. [7] analyzed the peristaltic flow and heat transfer in a vertical porous annulus with long wavelength approximation. Srinivas and Gayathri [8] studied the peristaltic transport of a Newtonian fluid in a vertical asymmetric channel with heat transfer and a porous medium. Vajravelu et al. [9] discussed the influence of heat transfer on the peristaltic transport of a Jeffrey fluid in a vertical porous stratum. Sreenadh et al. [10] investigated the peristaltic transport of micropolar fluid in an asymmetric channel with permeable walls. The peristaltic transport of a conducting Jeffrey fluid in an inclined asymmetric channel was studied by Vajravelu et al. [11].

Magnetohydrodynamics describes the dynamics of electrically conducting fluids. The mutual interaction between the fluid motion and magnetic field is the essential feature of the physical situation in the MHD fluid flow problems. MHD principles are useful in the design of heat exchangers, pumps, radar systems, power generation development of magnetic devices, cancer tumor treatment, hyperthermia, and blood reduction during surgeries. It is realized that the principles of magneto hydrodynamics find extensive applications in bioengineering and medical sciences. Hence several scientists analyzed the peristalsis with magnetic field effects (Mekheimer [12]; Hayat and Ali [13]; Kothandapani and Srinivas [14]; Noreen et al. [15]).

Most of the biofluids in nature are now accepted to behave like non-Newtonian fluids. The complex behavior of chyme in the small intestine is modeled through a Phan-Thien-Tanner (PTT) fluid model by Hakeem and Naby [16] and a good agreement is found between theoretical and experimental results. Motivated by the above studies, the peristaltic transport of conducting Phan-Thien-Tanner fluid in an asymmetric channel with a porous medium is investigated. The governing equations of the PTT model have been simplified and are solved by a perturbation technique. The expressions for the stream function, the temperature, the pressure gradient, and the pressure rise have been obtained. The effects of various parameters on the velocity, the temperature, the pressure rise, and the trapping phenomenon are discussed through graphs.

\section{Mathematical formulation}

We consider an incompressible Phan-Thien-Tanner fluid in an asymmetric channel with a porous medium of width $d_{1}+d_{2}$. Let $c$ be the speed by which sinusoidal wave trains propagate along the channel walls. Consider the rectangular coordinate system $(\bar{X}, \bar{Y})$ where the $\bar{X}-$ axis and $\bar{Y}-$ axis are taken parallel and transverse to the direction of wave propagation, respectively. The wall surfaces are modeled as $H_{i}, i=1,2\left(H_{1}\right.$ is the upper wall and $H_{2}$ is the lower wall)

$$
\bar{Y}=H_{1}=d_{1}+a_{1} \cos \left[\frac{2 \pi}{\lambda}(\bar{X}-c \bar{t})\right], \quad \bar{Y}=H_{2}=-d_{2}-b_{1} \cos \left[\frac{2 \pi}{\lambda}(\bar{X}-c \bar{t})+\phi\right]
$$


where $\phi$ is the phase difference varying in the range $0 \leq \phi \leq \pi, \phi=0$ corresponds to the symmetric channel with waves out of phase and $\phi=\pi$ with waves in phase. Also, $a_{1}, b_{1}, d_{1}, d_{2}$ and $\phi$ satisfy the condition $a_{1}^{2}+b_{1}^{2}+2 a_{1} b_{1} \cos \phi \leq\left(d_{1}+d_{2}\right)^{2}$ so that walls will not intersect each other.

The governing equations of the model are as follows:

Continuity equation

$$
\nabla \cdot \boldsymbol{V}=0 .
$$

Momentum equation

$$
\rho \cdot \frac{d \boldsymbol{V}}{d t}=\operatorname{div} \boldsymbol{T}
$$

The constitutive equations for the PTT model

$$
\begin{aligned}
& \boldsymbol{T}=-p \boldsymbol{I}+\boldsymbol{s}, \\
& f(\operatorname{tr}(\boldsymbol{s})) \boldsymbol{s}+k \boldsymbol{s}=2 \mu \boldsymbol{D}, \\
& \nabla \\
& \boldsymbol{s}=\frac{d \boldsymbol{s}}{d t}-\boldsymbol{s} \cdot L^{*}-\boldsymbol{L} . \boldsymbol{s}, \\
& \boldsymbol{L}=\operatorname{grad} \boldsymbol{V} ;
\end{aligned}
$$

asterisk denotes transposition.

The function $f$ in the linearized PTT model satisfies the expression

$$
f(\operatorname{tr}(s))=1+\frac{\varepsilon k}{\mu} \operatorname{tr}(s)
$$

Note that the PTT model reduces to an upper convected Maxwell (UCM) model when the extensional parameter $\varepsilon$ is zero.

We introduce the following transformations between the fixed and the wave frames

$$
\bar{x}=\bar{X}-c \bar{t}, \quad \bar{y}=\bar{Y}, \quad \bar{u}=\bar{U}-c, \quad \bar{v}=\bar{V}, \quad \bar{p}(\bar{x})=\bar{P}(\bar{X}, \bar{t}) .
$$

Using Eq.(2.9), the governing equations in the wave frame can be written as

$$
\begin{aligned}
& \frac{\partial \bar{u}}{\partial \bar{x}}+\frac{\partial \bar{v}}{\partial \bar{y}}=0, \\
& \rho\left[\bar{u} \frac{\partial}{\partial \bar{x}}+\bar{v} \frac{\partial}{\partial \bar{y}}\right] \bar{u}=-\frac{\partial \bar{p}}{\partial \bar{x}}+\frac{\partial \bar{S}_{x x}^{-}}{\partial \bar{x}}+\frac{\partial \bar{S}_{x \bar{y}}^{-}}{\partial \bar{y}}-\frac{\mu}{k_{0}}(\bar{u}+c)-\sigma_{e} B_{0}^{2}(\bar{u}+c),
\end{aligned}
$$




$$
\begin{aligned}
& \rho\left[\bar{u} \frac{\partial}{\partial \bar{x}}+\bar{v} \frac{\partial}{\partial \bar{y}}\right] \bar{v}=-\frac{\partial \bar{p}}{\partial \bar{y}}+\frac{\partial \bar{S}}{\partial \overline{y x}}+\frac{\partial \bar{S}}{\partial \bar{y}}-\frac{\mu}{k_{0}} \bar{v} \\
& f \bar{S}_{\overline{x x}}+k\left[\bar{u} \frac{\partial \bar{S}_{x x}}{\partial \bar{x}}+\bar{v} \frac{\partial \bar{S}_{x x}}{\partial \bar{y}}-2 \frac{\partial \bar{u}}{\partial \bar{x}} \bar{S}_{\overline{x x}}-2 \frac{\partial \bar{u}}{\partial \bar{y}} \bar{S}_{\overline{x y}}\right] \bar{v}=2 \mu \frac{\partial \bar{u}}{\partial \bar{x}} \\
& f \bar{S}_{x x}+k\left[\bar{u} \frac{\partial \bar{S}_{\overline{y y}}}{\partial \bar{x}}+\bar{v} \frac{\partial \bar{S}_{\overline{y y}}}{\partial \bar{y}}-2 \frac{\partial \bar{v}}{\partial \bar{x}} \bar{S}_{\overline{y x}}-2 \frac{\partial \bar{v}}{\partial \bar{y}} \bar{S}_{\overline{y y}}\right] \bar{v}=2 \mu \frac{\partial \bar{v}}{\partial \bar{y}} \\
& f \bar{S}_{z z}^{-}+k\left[\bar{u} \frac{\partial \bar{S}_{z z}}{\partial \bar{x}}+\bar{v} \frac{\partial \bar{S}}{\overline{z z}}\right]=0 \\
& f \bar{S}_{x y}+k\left[\bar{u} \frac{\partial \bar{S}_{x y}}{\partial \bar{x}}+\bar{v} \frac{\partial \bar{S}_{x y}^{-}}{\partial \bar{y}}-\frac{\partial \bar{v}}{\partial \bar{x}} \bar{S}_{x x}^{-}-\frac{\partial \bar{v}}{\partial \bar{y}} \bar{S}_{x y}-\frac{\partial \bar{u}}{\partial \bar{x}} \bar{S}_{x y}^{-}-\frac{\partial \bar{u}}{\partial \bar{y}} \bar{S}_{\overline{y y}}\right]=\mu\left(\frac{\partial \bar{u}}{\partial \bar{y}}+\frac{\partial \bar{v}}{\partial \bar{x}}\right) \\
& f=1+\frac{\varepsilon k}{\mu}\left(\bar{S}_{\overline{x x}}+\bar{S}_{\overline{y y}}+\bar{S}_{\overline{z z}}\right), \\
& \rho c_{p}\left[(\bar{u}+c) \frac{\partial}{\partial \bar{x}}+\bar{v} \frac{\partial}{\partial \bar{y}}\right] T=k_{0}\left(\frac{\partial^{2}}{\partial \bar{x}^{2}}+\frac{\partial^{2}}{\partial \bar{y}^{2}}\right) T+2 \mu\left[\left(\frac{\partial \bar{u}}{\partial \bar{x}}\right)^{2}+\left(\frac{\partial \bar{v}}{\partial \bar{y}}\right)^{2}\right]+\mu\left(\frac{\partial \bar{u}}{\partial \bar{y}}+\frac{\partial \bar{v}}{\partial \bar{x}}\right)^{2}
\end{aligned}
$$

The non-dimensional quantities and the expressions for stream functions are given by

$$
\begin{aligned}
& \mathrm{We}=\frac{k c}{d_{1}}, \quad x=\frac{\bar{x}}{\lambda}, \quad y=\frac{\bar{y}}{d_{1}}, u=\frac{\bar{u}}{c}, v=\frac{\bar{v}}{\delta c}, \delta=\frac{d_{1}}{\lambda}, \quad p=\frac{d_{1}^{2} \bar{p}}{\mu c \lambda}, t=\frac{c \bar{t}}{\lambda}, \\
& \operatorname{Re}=\frac{\rho c d_{1}}{\mu}, \quad d=\frac{d_{2}}{d_{1}}, \quad a=\frac{a_{1}}{d_{1}}, \quad b=\frac{b_{1}}{d_{1}}, \quad S_{i j}=\frac{\overline{S_{i j}} d_{1}}{\mu c}, \quad h_{1}=\frac{H_{1}}{d_{1}}=1+a \cos (2 \pi x), \\
& h_{2}=\frac{H_{2}}{d_{1}}=-d-b \cos (2 \pi x+\phi) M^{2}=\frac{\sigma_{e} B_{0}^{2} d_{1}^{2}}{\mu}, \quad \sigma=\frac{d_{1}}{\sqrt{k}}, \\
& \theta=\frac{T-T_{0}}{T_{1}-T_{0}}, \quad \operatorname{Pr}=\frac{\mu c_{p}}{k_{0}}, \quad \operatorname{Ec}=\frac{c^{2}}{c_{p}\left(T_{1}-T_{0}\right)} \text { and } \quad u=\frac{\partial \psi}{\partial y}, \quad v=-\frac{\partial \psi}{\partial x} .
\end{aligned}
$$
equations reduces to

Using the above non-dimensional quantities and the long wave length approximation, the basic 


$$
\begin{aligned}
& \frac{d p}{d x}=\frac{\partial S_{x y}}{\partial y}-\left(\sigma^{2}+M^{2}\right)\left(\frac{\partial \psi}{\partial y}+1\right) \\
& \frac{\partial p}{\partial y}=0 \\
& f S_{x x}=2 \mathrm{We} \frac{\partial^{2} \psi}{\partial y^{2}} S_{x y}, \\
& f S_{y y}=0, \quad f S_{z z}=0 \\
& f S_{x x}=-\mathrm{We} \frac{\partial^{2} \psi}{\partial y^{2}} S_{y y}+\frac{\partial^{2} \psi}{\partial y^{2}} \\
& 0=\frac{1}{\operatorname{Pr}} \frac{\partial^{2} \theta}{\partial y^{2}}+E\left(\frac{\partial \psi}{\partial y}\right)^{2}
\end{aligned}
$$

The corresponding non-dimensional boundary conditions are

$$
\begin{aligned}
& \psi=\frac{F}{2}, \quad \frac{\partial \psi}{\partial y}=-1 \quad \text { at } y=h_{1} . \\
& \psi=-\frac{F}{2}, \quad \frac{\partial \psi}{\partial y}=-1 \quad \text { at } y=h_{2}, \\
& \theta=0 \quad \text { at } y=h_{1}, \\
& \theta=1 \quad \text { at } y=h_{2}
\end{aligned}
$$

where $F$ is the mean flow rate in the wave frame.

The flux at any axial station in the fixed frame is

$$
Q=\int_{h_{2}}^{h_{1}}(u+1) d y=h_{1}-h_{2}+F .
$$

The average volume flow rate over one period of the peristaltic wave is defined as

$$
\Theta=\frac{1}{T} \int_{0}^{T} Q d t=\frac{1}{T} \int_{0}^{T}\left(h_{1}-h_{2}+F\right) d t=F+1+d .
$$


From Eq.(2.23) we have $S_{y y}=0, S_{z z}=0$ and from Eq.(2.20) we get

$$
S_{x y}=y \frac{d p}{d x}+\left(\sigma^{2}+M^{2}\right)(\psi+y)
$$

With the help of Eqs (2.23) and (2.24) we can write

$$
S_{x x}=2 \mathrm{We} S_{x y}^{2}
$$

From Eqs (2.17), (2.23) and (2.29) we obtain

$$
\frac{\partial^{2} \psi}{\partial y^{2}}=S_{x y}+2 \varepsilon \mathrm{We}^{2} S_{x y}^{3}
$$

Substituting Eq.(2.28) into Eq.(2.30), we get

$$
\frac{\partial^{2} \psi}{\partial y^{2}}=y \frac{d p}{d x}+\left(\sigma^{2}+M^{2}\right)(\psi+y)+2 \varepsilon \mathrm{We}^{2}\left(y \frac{d p}{d x}+\left(\sigma^{2}+M^{2}\right)(\psi+y)\right)^{3} .
$$

\section{Perturbation solution}

Equation (2.31) is non-linear, its exact solution is not possible, and hence we employ the perturbation technique to find the solution. For the perturbation solution, we expand the flow quantities in powers of the small parameter $\mathrm{We}^{2}$ as follows

$$
\begin{aligned}
& \psi=\psi_{0}+\mathrm{We}^{2} \psi_{1}+O\left(\mathrm{We}^{4}\right), \\
& F=F_{0}+\mathrm{We}^{2} F_{1}+O\left(\mathrm{We}^{4}\right), \\
& \phi=\phi_{0}+\mathrm{We}^{2} \phi_{1}+O\left(\mathrm{We}^{4}\right), \\
& \frac{d p}{d x}=\frac{d p_{0}}{d x}+\mathrm{We}^{2} \frac{d p_{1}}{d x}+O\left(\mathrm{We}^{4}\right), \\
& \theta=\theta_{0}+\mathrm{We}^{2} \theta_{1}+O\left(\mathrm{We}^{4}\right) .
\end{aligned}
$$
orders.

Using the above expressions in Eqs (2.25) - (2.31), we obtain a system of equations of different

\subsection{System of order $\mathrm{We}^{0}$}

The governing equations and boundary conditions of the zeroth-order are 


$$
\begin{aligned}
& \frac{\partial^{2} \psi_{0}}{\partial y^{2}}=y \frac{d p_{0}}{d x}+\left(\sigma^{2}+M^{2}\right)\left(\psi_{0}+y\right), \\
& 0=\frac{1}{\operatorname{Pr}} \frac{\partial^{2} \theta_{0}}{\partial y^{2}}+\operatorname{Ec}\left(\frac{\partial \psi_{0}}{\partial y}\right)^{2}, \\
& \psi_{0}=\frac{F_{0}}{2}, \quad \frac{\partial \psi_{0}}{\partial y}=-1 \quad \text { at } \quad y=h_{1}, \\
& \psi_{0}=-\frac{F_{0}}{2}, \quad \frac{\partial \psi_{0}}{\partial y}=-1 \quad \text { at } \quad y=h_{2}, \\
& \theta_{0}=0 \quad \text { at } \quad y=h_{1} . \\
& \theta_{0}=1 \quad \text { at } \quad y=h_{2} .
\end{aligned}
$$

The solution of the zeroth-order problem is given by

$$
\psi_{0}=c_{1} \cosh \sqrt{\sigma^{2}+M^{2}} y+c_{2} \sinh \sqrt{\sigma^{2}+M^{2}} y-y\left(\frac{1}{\left(\sigma^{2}+M^{2}\right)} \frac{d p_{0}}{d x}+1\right)
$$

Hence, the axial velocity and the temperature for the zeroth-order are

$$
\begin{aligned}
& u_{0}=\sqrt{\sigma^{2}+M^{2}}\left(c_{1} \sinh \sqrt{\sigma^{2}+M^{2}} y+c_{2} \cosh \sqrt{\sigma^{2}+M^{2}} y\right)-\left(\frac{1}{\left(\sigma^{2}+M^{2}\right)} \frac{d p_{0}}{d x}+1\right), \\
& \theta_{0}=-\left(\sigma^{2}+M^{2}\right)^{2} \operatorname{Br}\left(a_{1} \cosh 2 \sqrt{\sigma^{2}+M^{2}} y+a_{2} \sinh 2 \sqrt{\sigma^{2}+M^{2}} y+a_{3} y^{2}\right)+ \\
& +A_{1} y+A_{2} .
\end{aligned}
$$

\subsection{System of order $\mathrm{We}^{0}$}

The governing equations and boundary conditions of the first-order are

$$
\begin{aligned}
& \frac{\partial^{2} \psi_{1}}{\partial y^{2}}=y \frac{d p_{1}}{d x}+\left(\sigma^{2}+M^{2}\right) \psi_{1}+2 \varepsilon\left(y \frac{d p_{0}}{d x}+\left(\sigma^{2}+M^{2}\right)\left(\psi_{0}+y\right)\right)^{3}, \\
& 0=\frac{1}{\operatorname{Pr}} \frac{\partial^{2} \theta_{1}}{\partial y^{2}}+2 \operatorname{Ec} \frac{\partial^{2} \psi_{0}}{\partial y^{2}} \frac{\partial^{2} \psi_{1}}{\partial y^{2}},
\end{aligned}
$$




$$
\begin{aligned}
& \psi_{1}=\frac{F_{1}}{2}, \quad \frac{\partial \psi_{0}}{\partial y}=0 \quad \text { at } \quad y=h_{1}, \\
& \psi_{1}=-\frac{F_{1}}{2}, \quad \frac{\partial \psi_{0}}{\partial y}=0 \quad \text { at } y=h_{2}, \\
& \theta_{1}=0 \quad \text { at } y=h_{1}, \\
& \theta_{1}=0 \quad \text { at } y=h_{2} .
\end{aligned}
$$

The solution of the first-order problem is given by

$$
\begin{aligned}
& \psi_{1}=c_{3} \cosh \sqrt{\sigma^{2}+M^{2}} y+c_{4} \sinh \sqrt{\sigma^{2}+M^{2}} y-y\left(\frac{1}{\left(\sigma^{2}+M^{2}\right)} \frac{d p_{0}}{d x}+1\right)+ \\
& L_{31} y^{3}-L_{15} y^{2}+L_{32} y+\frac{1}{4}\left(L_{5} \cosh 3 \sqrt{\sigma^{2}+M^{2}} y+L_{6} \sinh 3 \sqrt{\sigma^{2}+M^{2}} y\right)+ \\
& +\cosh 2 \sqrt{\sigma^{2}+M^{2}} y\left(L_{34}+L_{33} y\right)+\sinh 2 \sqrt{\sigma^{2}+M^{2}} y\left(L_{36}+L_{35} y\right)+ \\
& +\sinh \sqrt{\sigma^{2}+M^{2}} y\left(L_{27}+L_{37} y-L_{38} y^{2}+L_{39} y^{3}-L_{40} y^{4}+L_{41} y^{5}-L_{42} y^{6}\right)+ \\
& +\cosh \sqrt{\sigma^{2}+M^{2}} y\left(L_{28}+L_{43} y-L_{44} y^{2}+L_{45} y^{3}-L_{46} y^{4}+L_{47} y^{5}-L_{48} y^{6}\right) .
\end{aligned}
$$

Hence, the corresponding first-order axial velocity and the temperature are

$$
\begin{aligned}
& u_{1}=\sqrt{\sigma^{2}+M^{2}}\left(c_{3} \sinh \sqrt{\sigma^{2}+M^{2}} y+c_{4} \cosh \sqrt{\sigma^{2}+M^{2}} y\right)-\frac{1}{\left(\sigma^{2}+M^{2}\right)} \frac{d p_{1}}{d x}+ \\
& +3 L_{31} y^{2}-2 L_{15} y+L_{32}+ \\
& +\frac{3 \sqrt{\sigma^{2}+M^{2}}}{4}\left(L_{5} \sinh 3 \sqrt{\sigma^{2}+M^{2}} y+L_{6} \cosh 3 \sqrt{\sigma^{2}+M^{2}} y\right)+ \\
& +\sinh 2 \sqrt{\sigma^{2}+M^{2}} y\left(L_{71}+2 \sqrt{\sigma^{2}+M^{2}} L_{33} y\right)+ \\
& +\cosh 2 \sqrt{\sigma^{2}+M^{2}} y\left(L_{72}+2 \sqrt{\sigma^{2}+M^{2}} L_{35} y\right)+ \\
& +\sinh \sqrt{\sigma^{2}+M^{2}} y\left(L_{73}+L_{74} y+L_{75} y^{2}+L_{76} y^{3}+L_{77} y^{4}+L_{78} y^{5}\right)+ \\
& +\cosh \sqrt{\sigma^{2}+M^{2}} y\left(L_{79}+L_{80} y+L_{81} y^{2}+L_{82} y^{3}+L_{83} y^{4}+L_{84} y^{5}\right),
\end{aligned}
$$




$$
\theta_{1}=-2 \operatorname{Ec} \operatorname{Pr}\left(\sigma^{2}+M^{2}\right)\left\{\begin{array}{l}
\cosh \sqrt{\sigma^{2}+M^{2}} y\left(k_{28}+y k_{26}\right)+\sinh \sqrt{\sigma^{2}+M^{2}} y\left(k_{29}+y k_{27}\right)+ \\
+\cosh 2 \sqrt{\sigma^{2}+M^{2}} y\left(k_{30}+k_{31} y+k_{32} y^{2}+k_{33} y^{3}+k_{34} y^{4}+k_{11} y^{5}\right)+ \\
+\sinh 2 \sqrt{\sigma^{2}+M^{2}} y\left(k_{35}+k_{36} y+k_{37} y^{2}+k_{38} y^{3}+k_{39} y^{4}+k_{12} y^{5}\right)+ \\
\cosh 3 \sqrt{\sigma^{2}+M^{2}} y\left(k_{40}+k_{41} y\right)+\sinh 3 \sqrt{\sigma^{2}+M^{2}} y\left(k_{42}+k_{43} y\right)+ \\
+k_{18} \cosh 4 \sqrt{\sigma^{2}+M^{2}} y+k_{19} \sinh 4 \sqrt{\sigma^{2}+M^{2}} y+k_{20} y^{2}+k_{21} y^{3}+ \\
+k_{22} y^{4}+k_{23} y^{5}+k_{24} y^{6}+k_{25} y^{7}
\end{array}\right\}+
$$

$+A_{3} y+A_{4}$.

Finally, the expressions for the axial velocity and the temperature are given by

$$
u=u_{0}+\mathrm{We}^{2} u_{1}
$$

and

$$
\theta=\theta_{0}+\mathrm{We}^{2} \theta_{1}
$$

The pressure gradient is obtained as

$$
\frac{d p}{d x}=\frac{d p_{0}}{d x}+\mathrm{We}^{2} \frac{d p_{1}}{d x} .
$$

Here

$$
\frac{d p_{0}}{d x}=\left(\sigma^{2}+M^{2}\right)\left(\frac{c_{1} L_{2}+c_{2} L_{3}-F_{0}}{h_{1}-h_{2}}-1\right) \quad \text { and } \quad \frac{d p_{1}}{d x}=\frac{F_{1}-L_{132}}{L_{131}} \text {. }
$$

The non-dimensional pressure rise per unit wave length in the wave frame is given by

$$
\Delta p=\int_{0}^{1} \frac{d p}{d x} d x .
$$

\section{Results and discussion}

The expression for the velocity field in terms of $y$ is given by Eq.(3.14). Velocity profiles are plotted in Figs $1-7$ to study the effects of different parameters such as the permeability parameter $\sigma$, the magnetic parameter $M$, the Weissenberg number We, the phase difference $\phi$ and the amplitudes $a, b$ on the velocity distribution. Figures 1 and 2 are drawn to study the effect of $\sigma$ and $M$. We notice that the velocity profiles are parabolic. Further we observe that the velocity decreases with increasing $\sigma$ and $M$. From Figs 3and 4, we notice that the velocity increases with an increase in We and $\phi$. Figure 5 reveals that the velocity increases 
with increasing $a$. From Fig.6,we noticed that the velocity decreases with increasing $b$. Figure7 depicts that the velocity decreases with an increase in $d$.

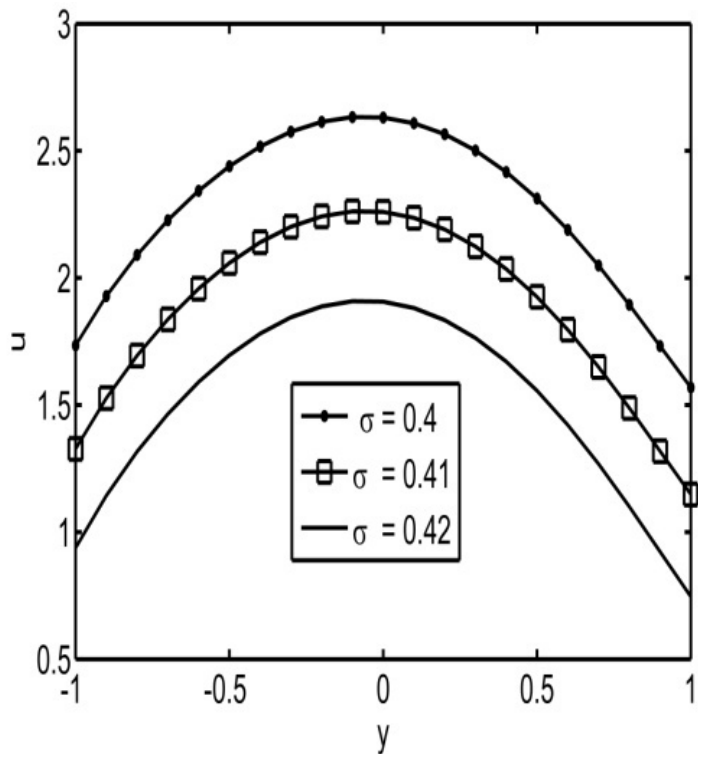

Fig.1. Velocity profiles for different ' $\sigma$ ' with Fig.2. Velocity profiles for different ' $\mathrm{M}$ ' with fixed: $a=0.5, b=0.5, d=1.2, x=0.1, \phi=6$, $M=0.6, F=1.5, \mathrm{We}=0.2$.

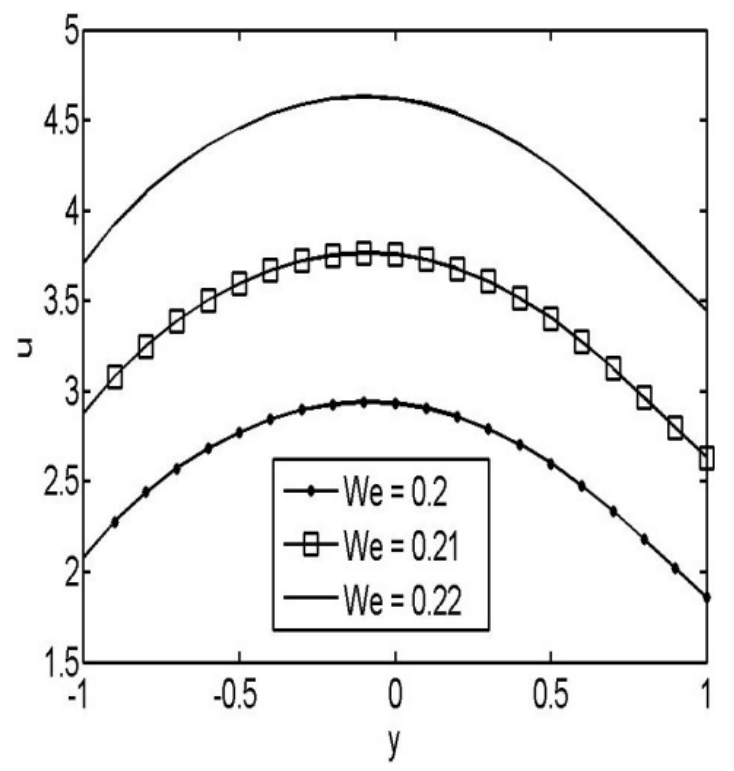

Fig.3. Velocity profiles for different 'We' with fixed: $a=0.5, b=0.5, d=1.2, x=0.1, \phi=\pi / 6$, $F=1.5, M=0.6, \sigma=0.4$.

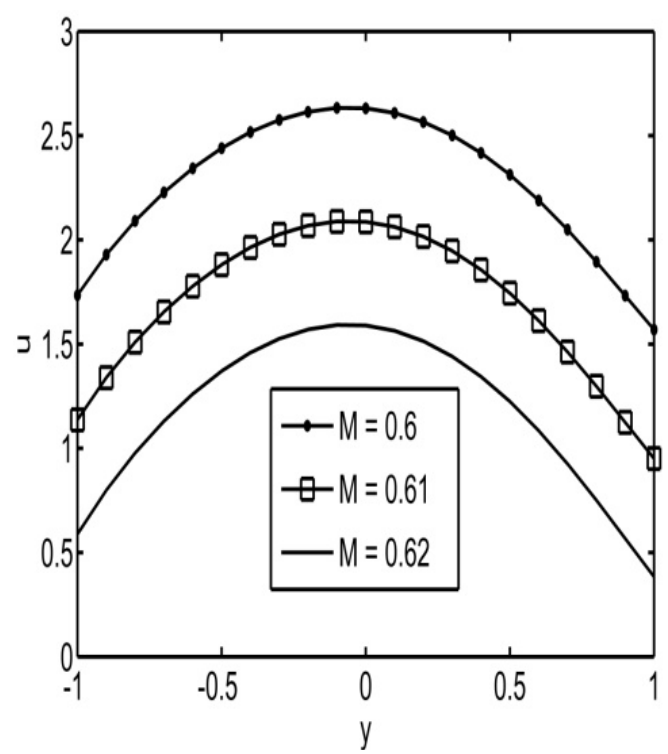

fixed: $a=0.5, b=0.5, d=1.2, x=0.1, \phi=\pi / 6$, $F=1.5, \sigma=0.4, \mathrm{We}=0.2$.

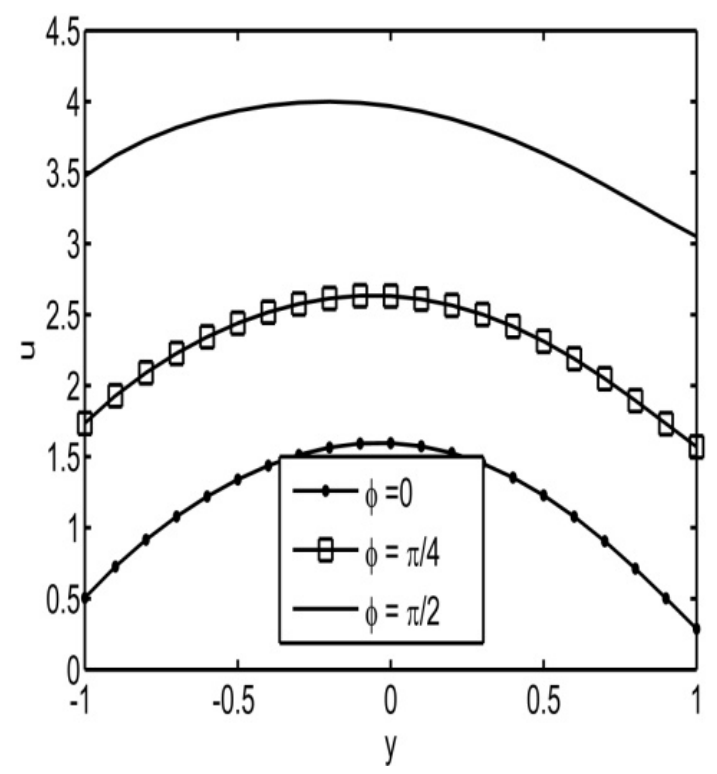

Fig.4. Velocity profiles for different ' $\phi$ ' with fixed: $a=0.5, b=0.5, d=1.2, x=0.1, M=0.6$, $\mathrm{We}=0.2, \sigma=0.4, F=1.5$. 


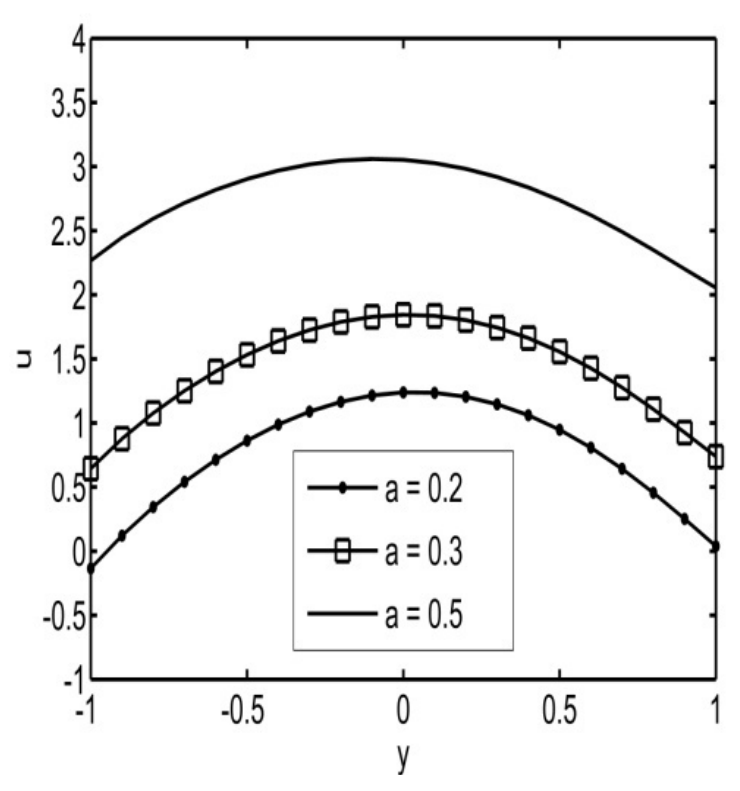

Fig.5. Velocity profiles for different ' $a$ ' with fixed: $b=0.5, \quad d=1.2, \quad x=0.1, \quad M=0.6, \quad \phi=\pi / 6$, $\mathrm{We}=0.2, \sigma=0.4, F=1.5$.

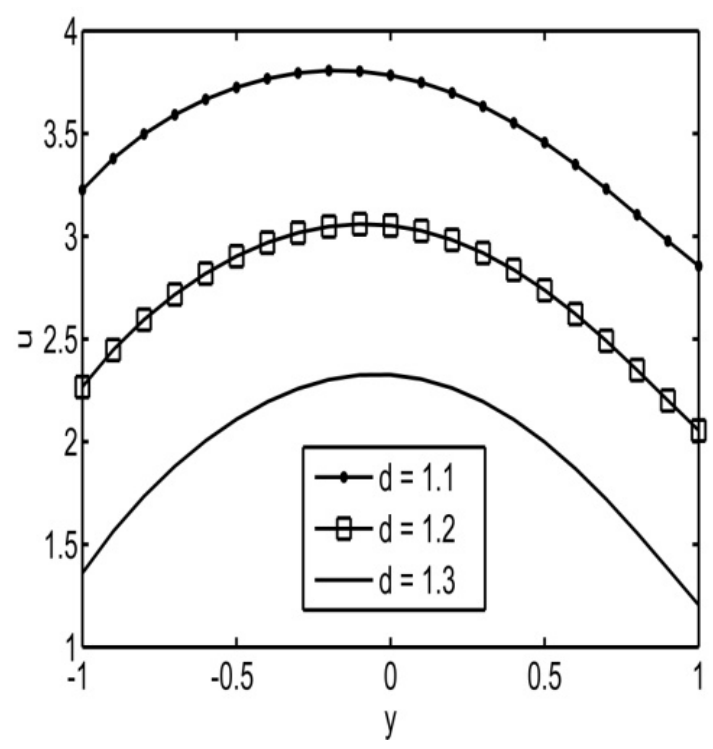

Fig.7. Velocity profiles for different ' $d$ ' with fixed: Fig.8. Temperature profiles for different ' $\sigma$ ' with $a=0.5, \quad b=0.5, \quad x=0.1, \quad M=0.6, \quad \phi=\pi / 6$, $\mathrm{We}=0.2, \sigma=0.4, F=1.5$.

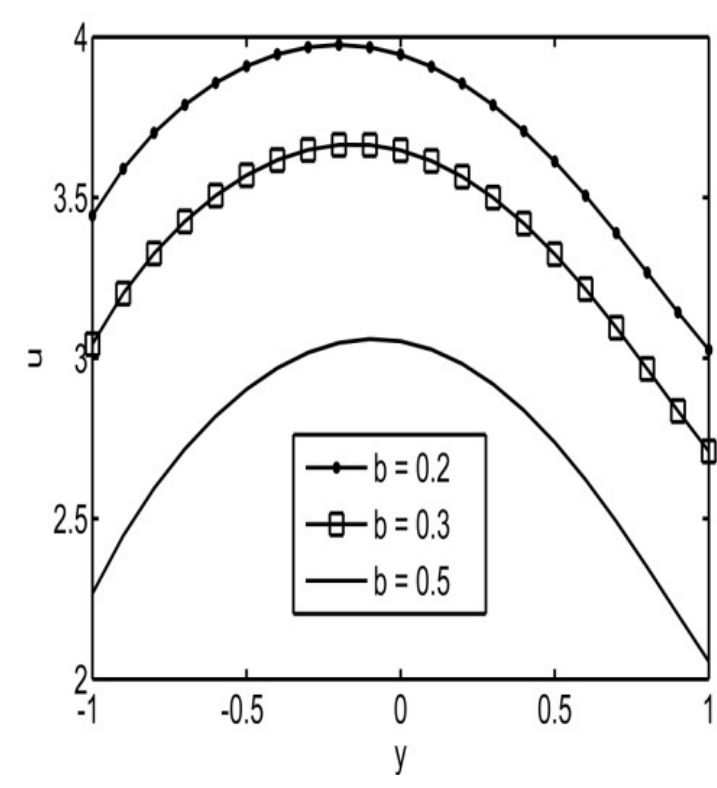

Fig.6. Velocity profiles for different ' $b$ ' with fixed: $a=0.5, \quad d=1.2, \quad x=0.1, \quad M=0.6, \quad \phi=\pi / 6$, $\mathrm{We}=0.2, \sigma=0.4, F=1.5$.

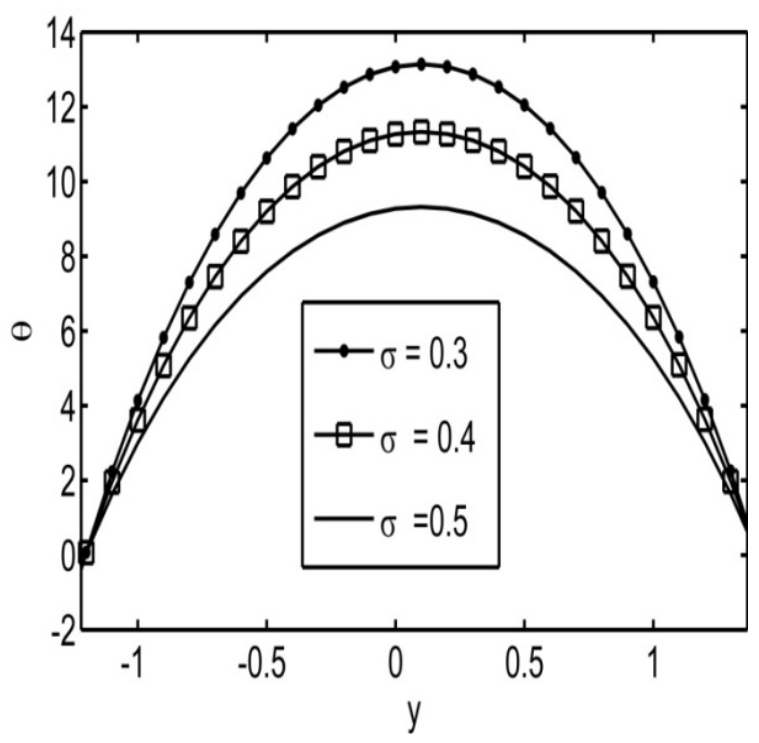

fixed: $a=0.5, b=0.5, d=1, x=0.1, \phi=\pi / 6$, $M=0.6, \mathrm{Br}=2$. 


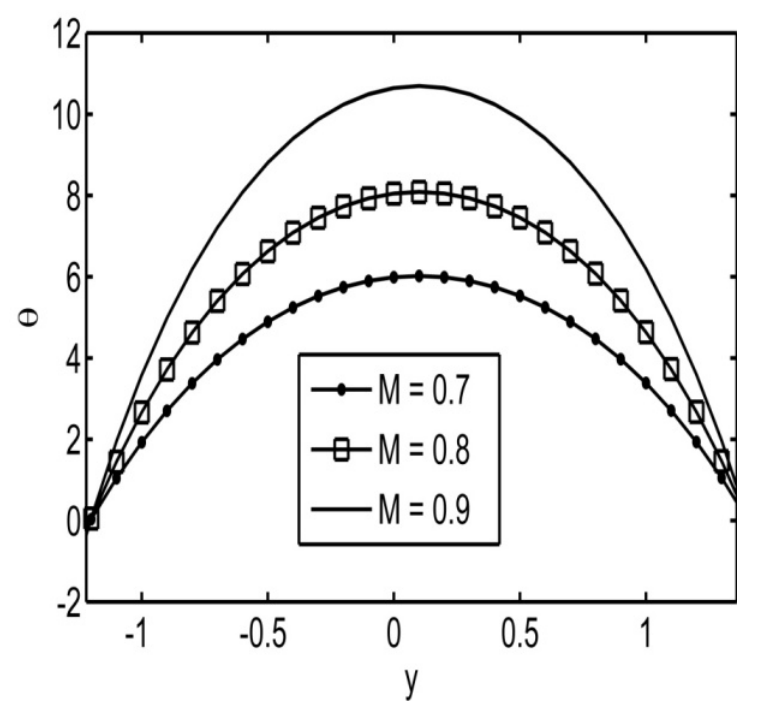

Fig.9. Temperature profiles for different ' $M$ ' with fixed: $a=0.5, b=0.5, d=1, x=0.1, \phi=\pi / 6$, $\sigma=0.2, \mathrm{Br}=2$.

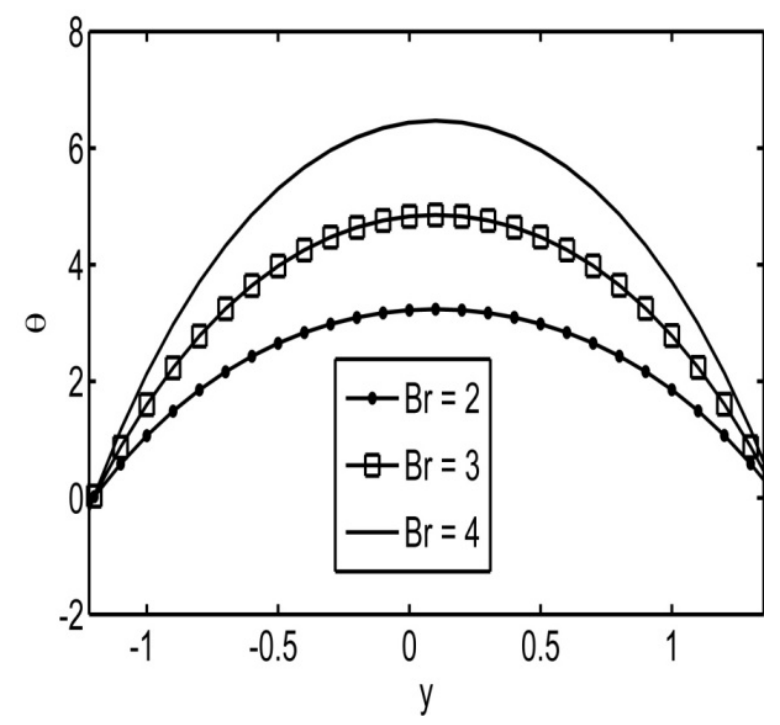

Fig.11. Temperature profiles for different ' $\mathrm{Br}$ ' with fixed: $a=0.5, b=0.5, d=1, x=0.1, \phi=\pi / 6$, $M=0.8, \sigma=0.2$.

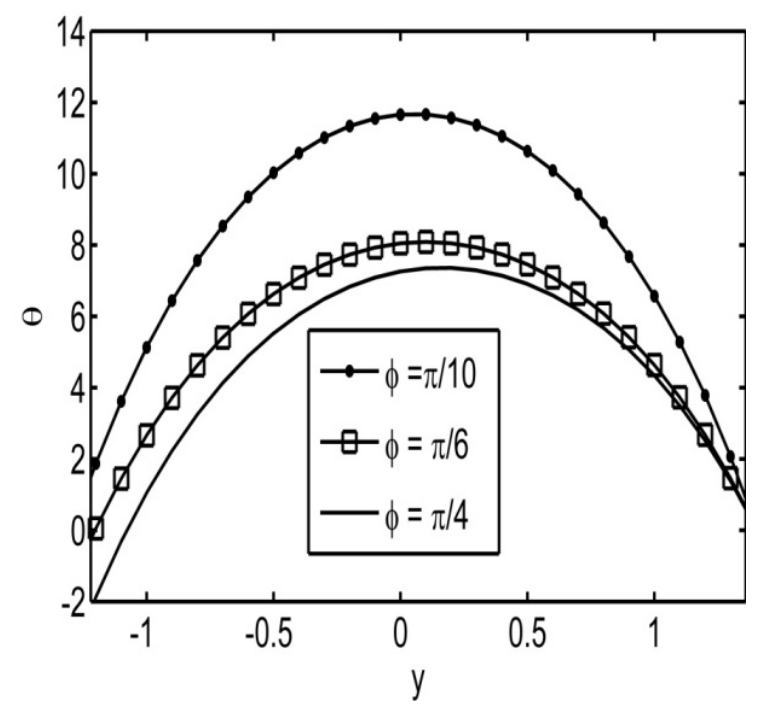

Fig.10. Temperature profiles for different ' $\phi$ ' with fixed: $a=0.5, b=0.5, d=1, x=0.1, \quad M=0.8$, $\sigma=0.2, \mathrm{Br}=2$.

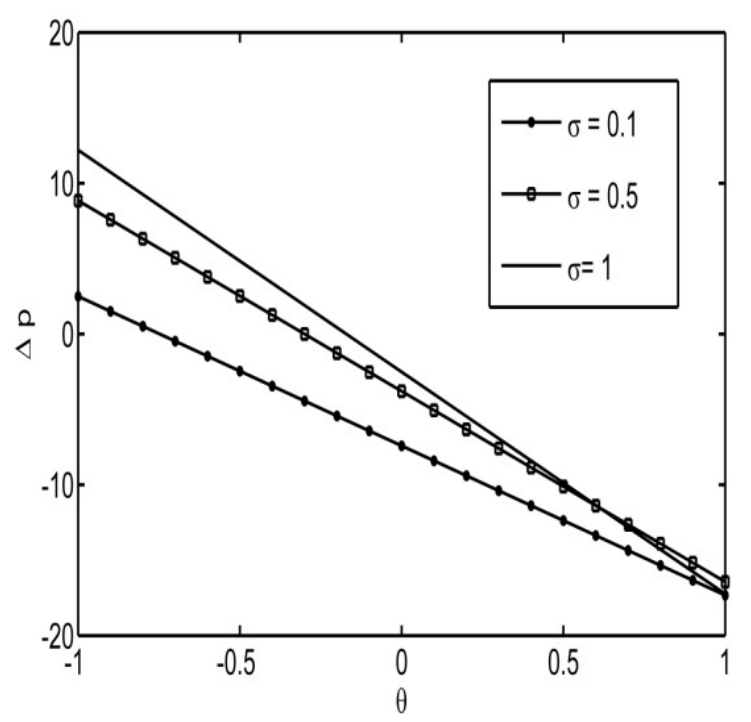

Fig.12. Variation of pressure rise for different ' $\sigma$ ' with fixed: $a=0.4, b=0.4, d=1, \phi=\pi / 8$, $M=0.5, \mathrm{We}=0.01$. 


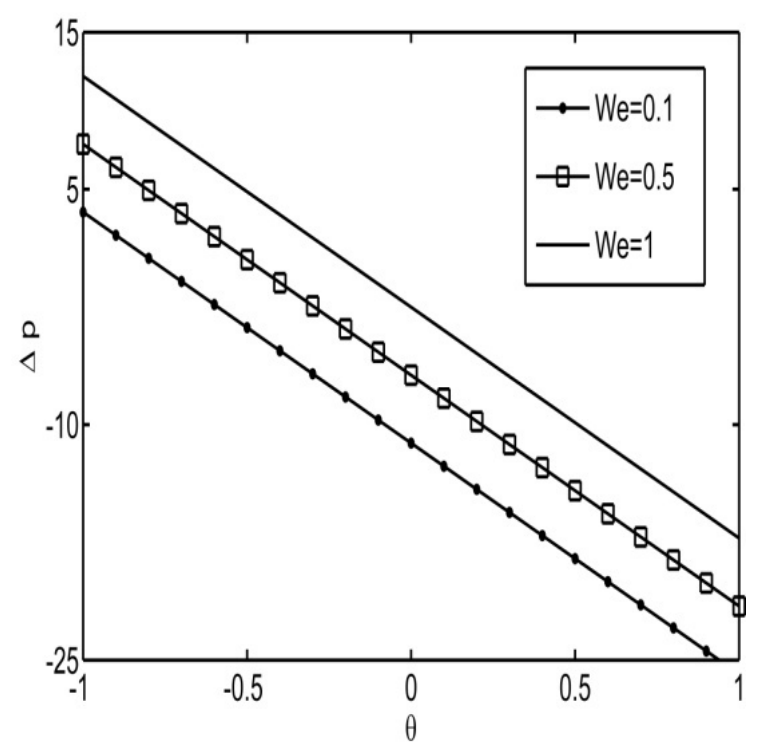

Fig.13. Variation of pressure rise for different 'We' with fixed: $a=0.4, b=0.4, d=1, M=0.5$, $\phi=\pi / 8, \sigma=0.1$

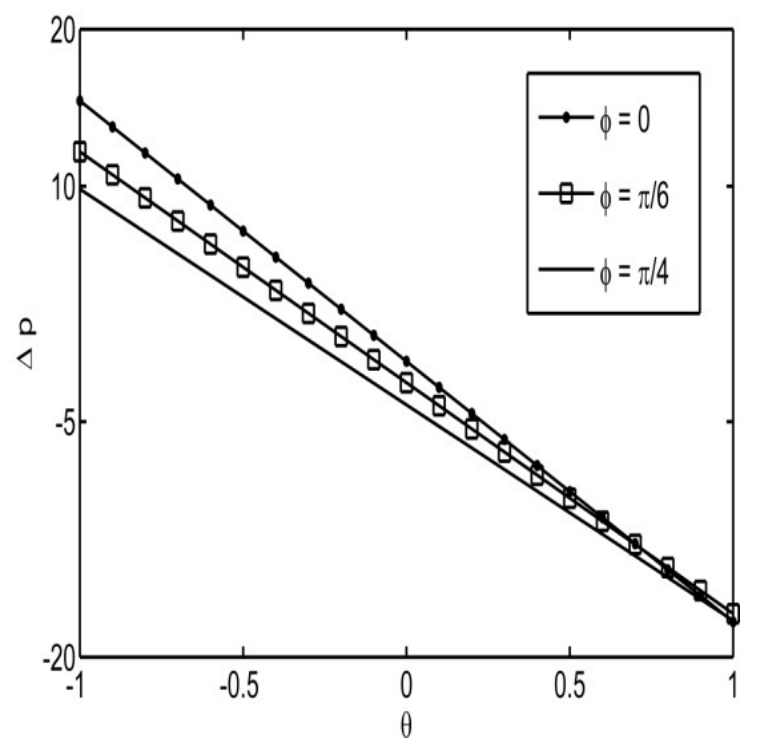

Fig.14. Variation of pressure rise for different ' $\phi$ ' with fixed: $a=0.4, b=0.4, d=1, \mathrm{We}=0.02$, $M=0.5, \sigma=0.1$.

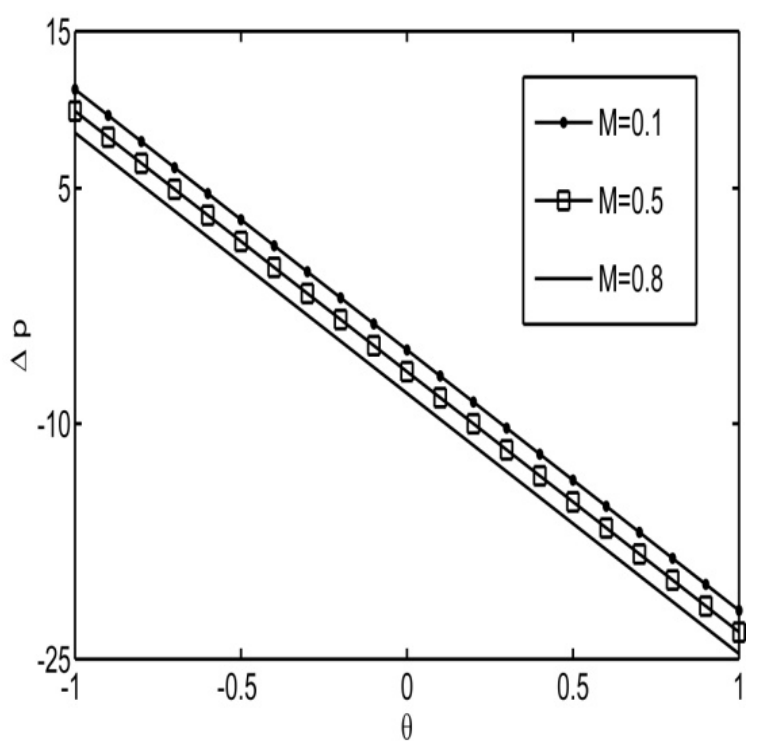

Fig.15. Variation of pressure rise for different ' $M$ ' with fixed: $a=0.4, b=0.4, d=1, \mathrm{We}=0.02, \sigma=0.1$, $\phi=\pi / 8$.

Equation (3.15) gives the expression for the temperature field in terms of $y$. Temperature profiles are plotted in Fig. 8 - Fig.11 to study the effects of the permeability parameter $\sigma$, the magnetic parameter $M$, the phase difference $\phi$, and the Brinkman number Br. Figure 8 is drawn to study the effect of $\sigma$ on the temperature. It is observed that the temperature profiles are parabolic and the temperature decreases with increasing permeability parameter $\sigma$. 
The influence of the magnetic parameter $M$ on the velocity is shown in Fig.9. We notice that the temperature increases with an increase in $M$. Figures 10 and 11 are plotted for different values of $\phi$ and $\mathrm{Br}$. It is noticed that the temperature increases with decreasing $\phi$ and an increase in $\mathrm{Br}$.

We have calculated the pressure rise $\Delta p$ in terms of the mean flow $\Theta$ from Eq.(3.17). Figure 12 shows the effect of $\sigma$ on $\Delta p$. We observe that for a given $\Theta$, the pressure rise decreases with increasing $\sigma$. The effect of We is shown in Fig.13. It can be seen that the pressure rise increases with an increase in We From Fig.14, we observe that the pressure rise decreases with increasing $\phi$. From Fig.15, we notice that the pressure rise decreases with an increase in $M$.

\section{Trapping phenomena}

The formation of an internally circulating bolus of fluid by closed streamlines is called trapping, and this trapped bolus is pushed ahead with the peristaltic wave. The effects of $\sigma, M$ and $\phi$ on the streamlines are shown in Fig.16- Fig.18. It is observed that the size of the trapping bolus decreases with increasing $\sigma$ and $M$. Also, it is noticed that the bolus increases with increasing $\phi$.
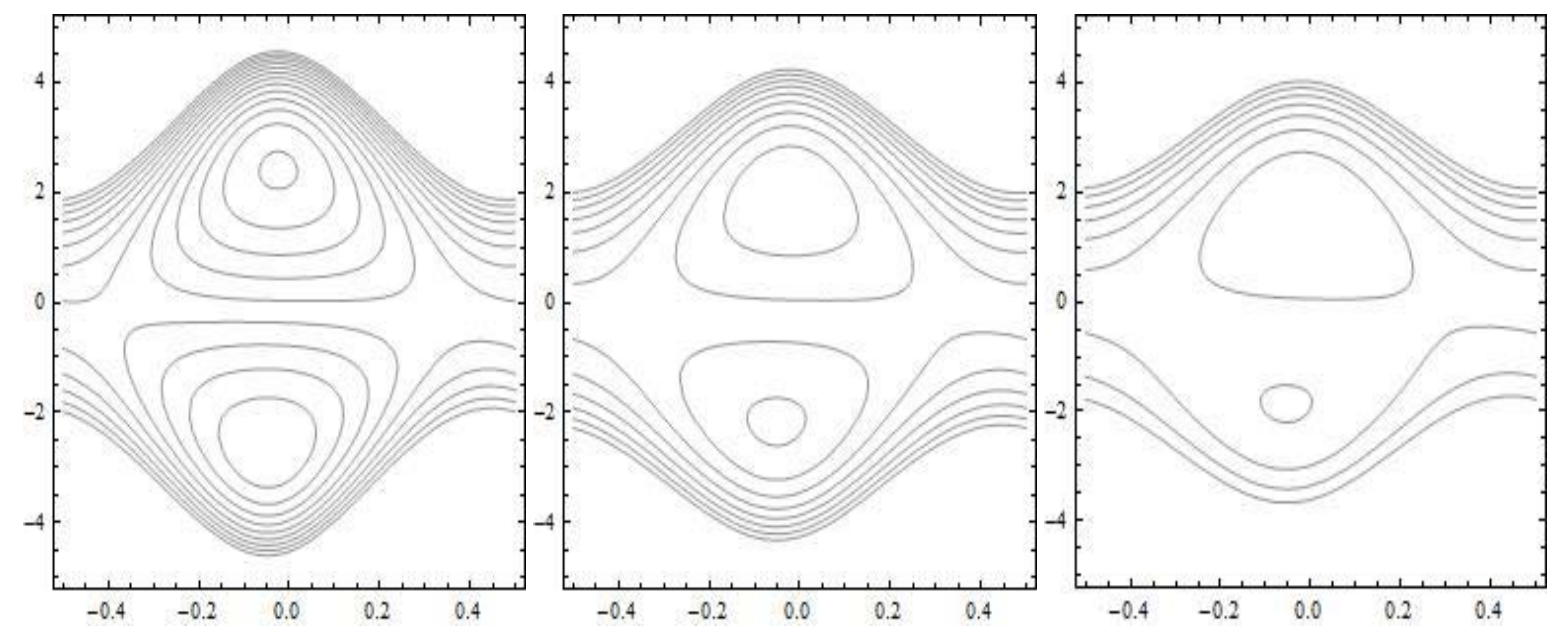

Fig.16. Stream lines for $a=0.4, b=0.3, d=1.3, \phi=\pi / 6, \mathrm{We}=0.01, F=10, M=0.6$ and for different values of $\sigma:$ (a) $\sigma=0.8$, (b) $\sigma=0.9$, (c) $\sigma=1$.
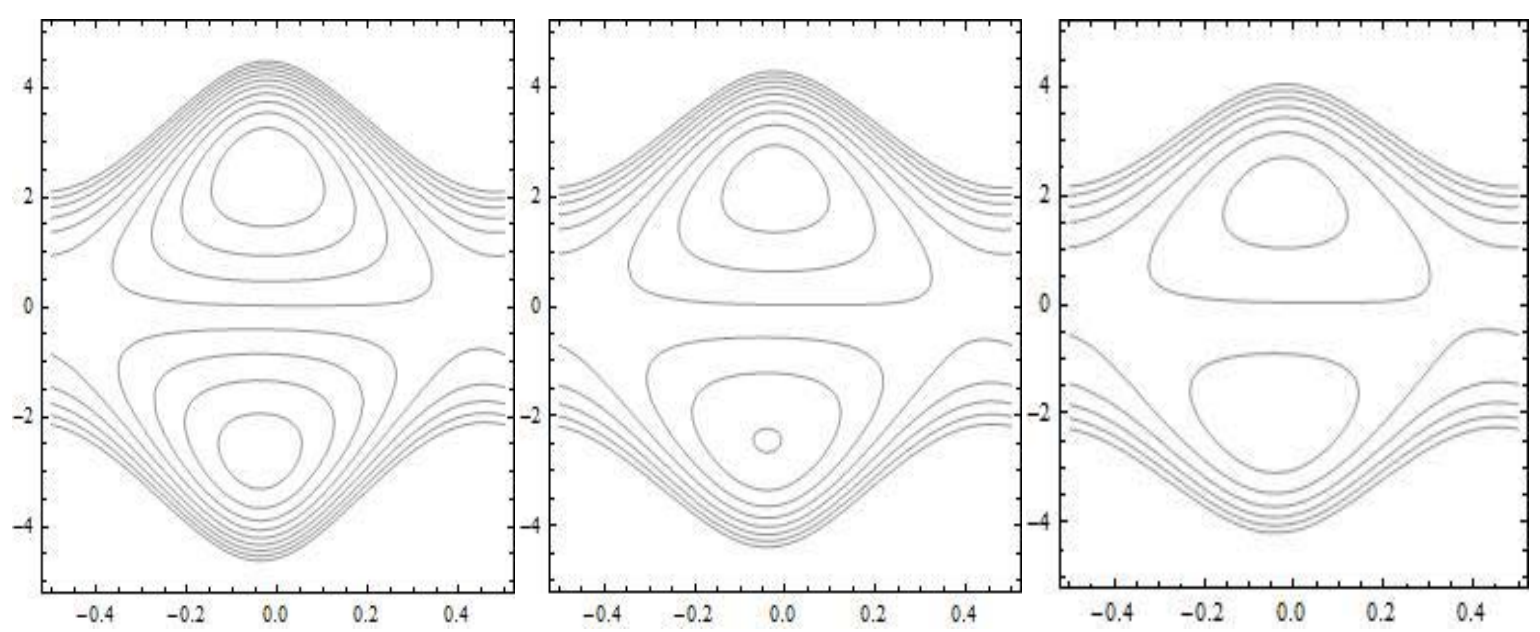

Fig.17. Stream lines for $a=0.3, b=0.3, d=1.2, \sigma=0.86, \mathrm{We}=0.001, F=8$, and for different values of $M$ : (a) $M=0.2$, (b) $M=0.3$, (c) $M=0.4$. 

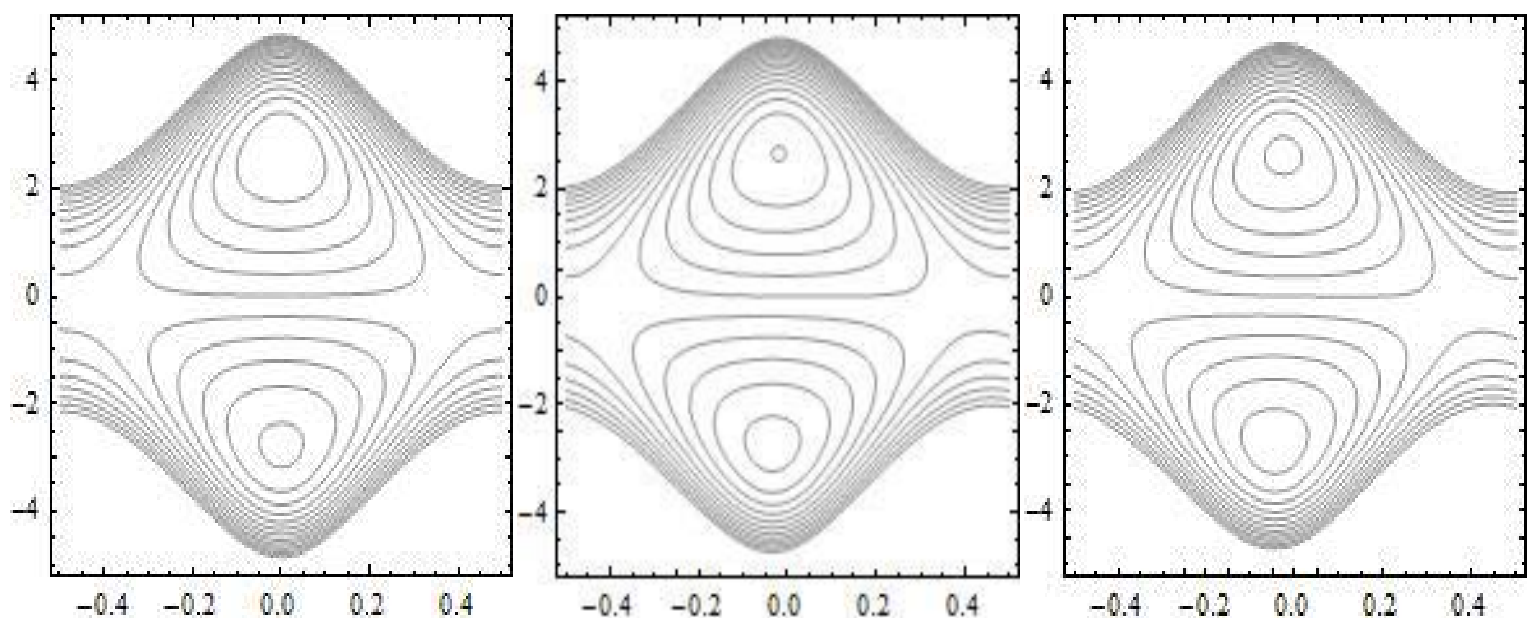

Fig.18. Stream lines for $a=0.4, b=0.3, d=1.2, \sigma=0.87, \mathrm{We}=0.001, F=8, M=0.2$ and for different values of $\phi:$ (a) $\phi=0$, (b) $\phi=\pi / 8$, (c) $\phi=\pi / 6$.

\section{Nomenclature}

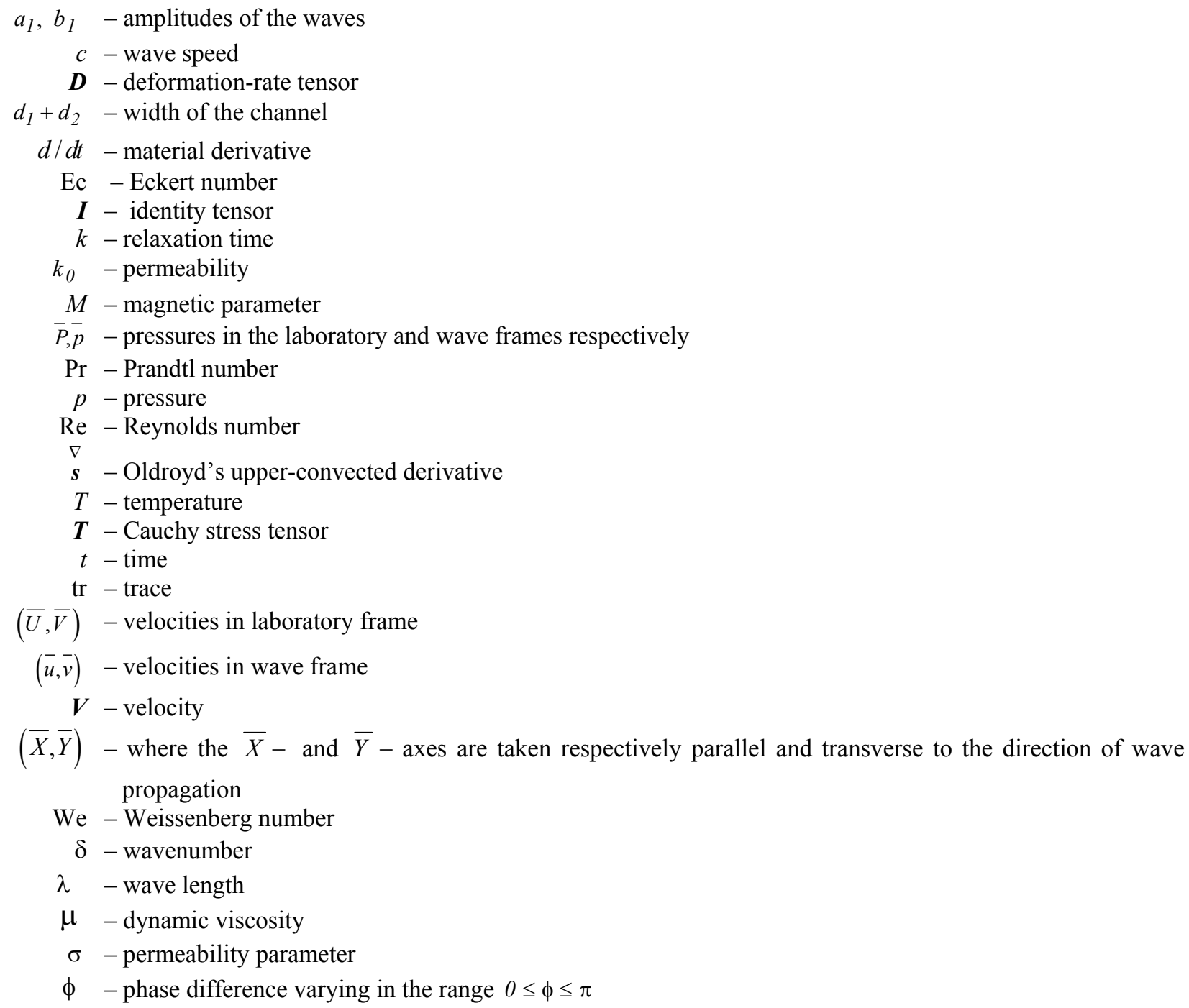




\section{Appendix}

$$
\begin{aligned}
& L_{1}=\frac{1}{\sigma^{2}+M^{2}} \frac{d p_{0}}{d x}+1, \quad L_{2}=\cosh \sqrt{\sigma^{2}+M^{2}} h_{1}-\cosh \sqrt{\sigma^{2}+M^{2}} h_{2}, \\
& L_{3}=\sinh \sqrt{\sigma^{2}+M^{2}} h_{1}-\sinh \sqrt{\sigma^{2}+M^{2}} h_{2}, \quad L_{2}^{l}=\cosh \sqrt{\sigma^{2}+M^{2}} h_{1}+\cosh \sqrt{\sigma^{2}+M^{2}} h_{2}, \\
& L_{3}^{1}=\sinh \sqrt{\sigma^{2}+M^{2}} h_{1}+\sinh \sqrt{\sigma^{2}+M^{2}} h_{2}, \quad L_{4}^{1}=\left(\frac{\left(h_{1}+h_{2}\right)\left(L_{3}^{2}-L_{2}^{2}\right)}{\left(L_{3} L_{3}^{1}-L_{2} L_{2}^{1}\right)}+\left(h_{2}-h_{1}\right)\right), \\
& c_{1}=\frac{-c_{2} L_{2}}{L_{3}}, \quad c_{2}=\frac{L_{1} L_{3}\left(h_{2}+h_{1}\right)}{L_{3} L_{3}^{1}-L_{2} L_{2}^{l}}, \quad L_{4}=\left(\frac{d p_{0}}{d x}\right)^{3}+3\left(\sigma^{2}+M^{2}\right)\left(\frac{d p_{0}}{d x}\right)^{2} \\
& L_{5}=\left(\frac{c_{1}}{2}\right)^{3}+3\left(\frac{c_{1}}{2}\right)\left(\frac{c_{2}}{2}\right)^{2}, \quad L_{6}=\left(\frac{c_{2}}{2}\right)^{3}+3\left(\frac{c_{1}}{2}\right)^{2}\left(\frac{c_{2}}{2}\right), \quad L_{7}=\left(\frac{c_{1}}{2}\right)^{3}-\left(\frac{c_{1}}{2}\right)\left(\frac{c_{2}}{2}\right)^{2} \\
& L_{8}=\left(\frac{c_{2}}{2}\right)^{3}-\left(\frac{c_{1}}{2}\right)^{2}\left(\frac{c_{2}}{2}\right), \quad L_{9}=\left(\frac{c_{1}}{2}\right)^{2}-\left(\frac{c_{2}}{2}\right)^{2}, \quad L_{10}=\left(\frac{c_{2}}{2}+\frac{c_{1}}{2}\right) \frac{3 L_{1}^{2} \sqrt{\sigma^{2}+M^{2}}}{2}, \\
& L_{11}=\frac{3 L_{1}^{2} \sqrt{\sigma^{2}+M^{2}}}{2}\left(\frac{c_{1}}{2}-\frac{c_{2}}{2}\right), \quad L_{12}=\left(\frac{c_{2}}{2}\right)^{2}+\left(\frac{c_{1}}{2}\right)^{2}, \quad L_{13}=2\left(\frac{c_{2}}{2}\right)\left(\frac{c_{1}}{2}\right), \\
& L_{14}=\frac{L_{10}-L_{11}}{3}+L_{1}^{3}, \quad L_{15}=\frac{L_{10}+L_{11}}{2 \sqrt{\sigma^{2}+M^{2}}}, \quad L_{16}=3 L_{1} L_{9}+\frac{6 L_{1}^{3}}{\sigma^{2}+M^{2}}+\frac{L_{10}-L_{11}}{2\left(\sigma^{2}+M^{2}\right)}, \\
& L_{17}=3\left(\sigma^{2}+M^{2}\right)^{2}\left(\left(\sigma^{2}+M^{2}\right)+\frac{d p_{0}}{d x}\right), \quad L_{18}=\frac{6 L_{1}^{2}}{\sigma^{2}+M^{2}}+2 L_{9}, \quad L_{19}=\frac{2 L_{12} L_{17}}{3\left(\sigma^{2}+M^{2}\right)} \\
& L_{20}=\frac{L_{17} c_{1} c_{2}}{3\left(\sigma^{2}+M^{2}\right)}, \quad L_{21}=\frac{8 L_{12} L_{17}}{9\left(\sigma^{2}+M^{2}\right)^{3 / 2}}, \quad L_{22}=\frac{4 c_{1} c_{2} L_{17}}{9\left(\sigma^{2}+M^{2}\right)^{3 / 2}}, \quad L_{23}=\frac{L_{17} L_{8}}{\left(\sigma^{2}+M^{2}\right)}, \\
& L_{24}=\frac{L_{1}^{2} L_{17}}{\left(\sigma^{2}+M^{2}\right)}, \quad L_{25}=\frac{\left(c_{1}+c_{2}\right) L_{1} L_{17}}{3 \sqrt{\sigma^{2}+M^{2}}}, \quad L_{26}=\frac{\left(c_{1}+c_{2}\right) L_{1} L_{17}}{2\left(\sigma^{2}+M^{2}\right)^{3 / 2}}, \\
& L_{27}=\frac{c_{2} L_{1} L_{17}}{2\left(\sigma^{2}+M^{2}\right)}, L_{28}=\frac{c_{1} L_{1} L_{17}}{2\left(\sigma^{2}+M^{2}\right)}, \quad L_{29}=3\left(\sigma^{2}+M^{2}\right)\left(1+\frac{2}{\left(\sigma^{2}+M^{2}\right)} \frac{d p_{0}}{d x}+\left(\frac{d p_{0}}{d x}\right)^{2}\right)
\end{aligned}
$$




$$
\begin{aligned}
& L_{30}=\frac{L_{29} L_{1}}{2 \sqrt{\sigma^{2}+M^{2}}}, \quad L_{31}=L_{14}-L_{24}-\frac{L_{4}}{\left(\sigma^{2}+M^{2}\right)}, \quad L_{32}=L_{16}-L_{23}-\frac{6 L_{4}}{\left(\sigma^{2}+M^{2}\right)^{2}}, \\
& L_{33}=L_{19}-2 L_{1} L_{12}, \quad L_{34}=\frac{8 L_{1} L_{13}}{3 \sqrt{\left(\sigma^{2}+M^{2}\right)}}-L_{22}, \quad L_{35}=L_{20}-2 L_{1} L_{13}, \\
& L_{36}=\frac{8 L_{1} L_{12}}{3 \sqrt{\left(\sigma^{2}+M^{2}\right)}}-L_{21}, \quad L_{37}=3 \sqrt{\left(\sigma^{2}+M^{2}\right)} L_{7}+15 \frac{L_{30} c_{2}}{4\left(\sigma^{2}+M^{2}\right)^{5 / 2}}, \\
& L_{38}=\frac{15 L_{30} c_{1}}{4\left(\sigma^{2}+M^{2}\right)^{2}}, \quad L_{39}=\frac{5 L_{30} c_{2}}{2\left(\sigma^{2}+M^{2}\right)^{3 / 2}}, \quad L_{40}=\frac{5 L_{30} c_{1}}{4\left(\sigma^{2}+M^{2}\right)}, \\
& L_{41}=\frac{L_{30} c_{2}}{2 \sqrt{\left(\sigma^{2}+M^{2}\right)}}, \quad L_{42}=\frac{L_{30} c_{1}}{6}, \quad L_{43}=\frac{15 L_{30} c_{1}}{4\left(\sigma^{2}+M^{2}\right)^{5 / 2}}-L_{26}-3 \sigma L_{8}, \\
& L_{44}=\frac{15 L_{30} c_{2}}{4\left(\sigma^{2}+M^{2}\right)^{2}}, \quad L_{45}=\frac{5 L_{30} c_{1}}{2\left(\sigma^{2}+M^{2}\right)^{3 / 2}}-L_{25}, \quad L_{46}=\frac{5 L_{30} c_{2}}{4\left(\sigma^{2}+M^{2}\right)}, \\
& L_{47}=\frac{L_{30} c_{1}}{2\left(\sigma^{2}+M^{2}\right)}, L_{48}=\frac{L_{30} c_{2}}{6}, L_{49}=L_{31} h_{1}^{3}-L_{15} h_{1}^{2}+L_{32} h_{1} \text {, } \\
& L_{50}=\frac{L_{5} \cosh 3 \sqrt{\left(\sigma^{2}+M^{2}\right)} h_{1}+L \sinh 3 \sqrt{\left(\sigma^{2}+M^{2}\right)} h_{1}}{4}, L_{51}=\cosh 2 \sqrt{\left(\sigma^{2}+M^{2}\right)} h_{l}\left(L_{34}+L_{33} h_{1}\right), \\
& L_{52}=\sinh 2 \sqrt{\left(\sigma^{2}+M^{2}\right)} h_{1}\left(L_{36}+L_{35} h_{1}\right), \quad L_{53}=L_{27}+L_{37} h_{1}-L_{38} h_{1}^{2}+L_{39} h_{1}^{3}, L_{54}=-L_{40} h_{1}^{4}+L_{41} h_{1}^{5}-L_{42} h_{1}^{6} \text {, } \\
& L_{55}=\sinh \sqrt{\left(\sigma^{2}+M^{2}\right)} h_{1}\left(L_{53}+L_{54}\right), L_{56}=L_{28}+L_{43} h_{1}-L_{44} h_{1}^{2}+L_{45} h_{1}^{3}, L_{57}=-L_{46} h_{1}^{4}+L_{47} h_{1}^{5}-L_{48} h_{1}^{6}, \\
& L_{58}=\cosh \sqrt{\left(\sigma^{2}+M^{2}\right)} h_{1}\left(L_{56}+L_{57}\right), \quad L_{59}=L_{49}+L_{50}+L_{51}+L_{52}+L_{55}+L_{58}, L_{60}=L_{32} h_{2}-L_{15} h_{2}^{2}+L_{31} h_{2}^{3} \text {, } \\
& L_{61}=\frac{L_{5} \cosh 3 \sqrt{\left(\sigma^{2}+M^{2}\right)} h_{2}+L_{6} \sinh 3 \sqrt{\left(\sigma^{2}+M^{2}\right)} h_{2}}{4}, \quad L_{62}=\cosh 2 \sqrt{\left(\sigma^{2}+M^{2}\right)} h_{2}\left(L_{34}+L_{33} h_{2}\right), \\
& L_{63}=\sinh 2 \sqrt{\left(\sigma^{2}+M^{2}\right)} h_{2}\left(L_{36}+L_{35} h_{2}\right), L_{64}=L_{27}+L_{37} h_{2}-L_{38} h_{2}^{2}+L_{39} h_{2}^{3}, L_{65}=-L_{40} h_{2}^{4}+L_{41} h_{2}^{5}-L_{42} h_{2}^{6} \text {, }
\end{aligned}
$$




$$
\begin{aligned}
& L_{66}=\sinh \sqrt{\left(\sigma^{2}+M^{2}\right)} h_{2}\left(L_{64}+L_{65}\right), L_{67}=L_{28} L_{43} h_{2}-L_{44} h_{2}^{2}+L_{45} h_{2}^{3}, L_{68}=-L_{46} h_{2}^{4}+L_{47} h_{2}^{5}-L_{48} h_{2}^{6}, \\
& L_{69}=\cosh \sqrt{\left(\sigma^{2}+M^{2}\right)} h_{2}\left(L_{67}+L_{68}\right), L_{70}=L_{60}+L_{61}+L_{62}+L_{63}+L_{66}+L_{69}, \quad L_{71}=2 \sqrt{\left(\sigma^{2}+M^{2}\right)} L_{34}+L_{35} \\
& L_{72}=L_{33}+2 \sqrt{\left(\sigma^{2}+M^{2}\right)} L_{36}, L_{73}=L_{37}+\sqrt{\left(\sigma^{2}+M^{2}\right)} L_{28}, L_{74}=\sqrt{\left(\sigma^{2}+M^{2}\right)} L_{43}-2 L_{38}, \\
& L_{75}=3 L_{39}-\sqrt{\left(\sigma^{2}+M^{2}\right)} L_{44}, \quad L_{76}=\sqrt{\left(\sigma^{2}+M^{2}\right)} L_{45}-4 L_{40}, L_{77}=5 L_{41}-\sqrt{\left(\sigma^{2}+M^{2}\right)} L_{46}, \\
& L_{78}=\sqrt{\left(\sigma^{2}+M^{2}\right)} L_{47}-6 L_{42}, \quad L_{79}=\sqrt{\left(\sigma^{2}+M^{2}\right)} L_{27}+L_{43}, L_{80}=\sqrt{\left(\sigma^{2}+M^{2}\right)} L_{37}-2 L_{44} \text {, } \\
& L_{81}=3 L_{45}-\sqrt{\left(\sigma^{2}+M^{2}\right)} L_{48} L_{77}, L_{82}=\sigma L_{39}-4 L_{46}, L_{83}=5 L_{47}-\sqrt{\left(\sigma^{2}+M^{2}\right)} L_{40}, \\
& L_{84}=\sqrt{\left(\sigma^{2}+M^{2}\right)} L_{41}-6 L_{48}, \quad L_{85}=3 L_{31} h_{1}^{2}-2 L_{15} h_{1}+L_{32}, \\
& L_{86}=3 \sigma\left(\frac{L_{5} \sinh 3 \sqrt{\left(\sigma^{2}+M^{2}\right)} h_{1}+L_{6} \cosh 3 \sqrt{\left(\sigma^{2}+M^{2}\right)} h_{1}}{4}\right) \text {, } \\
& L_{87}=\sinh 2 \sqrt{\left(\sigma^{2}+M^{2}\right)} h_{1}\left(L_{71}+2 \sqrt{\left(\sigma^{2}+M^{2}\right)} L_{33} h_{1}\right), L_{88}=\cosh 2 \sqrt{\left(\sigma^{2}+M^{2}\right)} h_{1}\left(L_{72}+2 \sqrt{\left(\sigma^{2}+M^{2}\right)} L_{35} h_{1}\right), \\
& L_{89}=L_{73}+L_{74} h_{1}+L_{75} h_{1}^{2}, \quad L_{90}=L_{76} h_{1}^{3}+L_{77} h_{1}^{4}+L_{78} h_{1}^{5}, \quad L_{91}=\sinh \sqrt{\left(\sigma^{2}+M^{2}\right)} h_{1}\left(L_{89}+L_{90}\right), \\
& L_{92}=L_{79}+L_{80} h_{1}+L_{81} h_{1}^{2}, \quad L_{93}=L_{82} h_{1}^{3}+L_{83} h_{1}^{4}+L_{84} h_{1}^{5}, \quad L_{94}=\cosh \sqrt{\left(\sigma^{2}+M^{2}\right)} h_{1}\left(L_{92}+L_{93}\right), \\
& L_{95}=L_{85}+L_{86}+L_{87}+L_{88}+L_{91}+L_{94}, \quad L_{96}=3 L_{31} h_{2}^{2}-2 L_{15} h_{2}+L_{32}, \\
& L_{97}=3 \sqrt{\left(\sigma^{2}+M^{2}\right)}\left(\frac{L_{5} \sinh 3 \sqrt{\left(\sigma^{2}+M^{2}\right)} h_{2}+L_{6} \cosh 3 \sqrt{\left(\sigma^{2}+M^{2}\right)} h_{2}}{4}\right),
\end{aligned}
$$




$$
\begin{aligned}
& L_{98}=\sinh 2 \sqrt{\left(\sigma^{2}+M^{2}\right)} h_{2}\left(L_{71}+2 \sqrt{\left(\sigma^{2}+M^{2}\right)} L_{33} h_{2}\right), L_{99}=\cosh 2 \sqrt{\left(\sigma^{2}+M^{2}\right)} h_{2}\left(L_{72}+2 \sqrt{\left(\sigma^{2}+M^{2}\right)} L_{35} h_{2}\right), \\
& L_{100}=L_{73}+L_{74} h_{2}+L_{75} h_{2}^{2}, L_{101}=L_{76} h_{2}^{3}+L_{77} h_{2}^{4}+L_{78} h_{2}^{5}, L_{102}=\sinh \sqrt{\left(\sigma^{2}+M^{2}\right)} h_{2}\left(L_{100}+L_{101}\right), \\
& L_{103}=L_{79}+L_{80} h_{2}+L_{81} h_{2}^{2}, L_{104}=L_{82} h_{2}^{3}+L_{83} h_{2}^{4}+L_{84} h_{2}^{5}, L_{105}=\cosh \sqrt{\left(\sigma^{2}+M^{2}\right)} h_{2}\left(L_{103}+L_{104}\right), \\
& L_{106}=L_{96}+L_{97}+L_{98}+L_{99}+L_{102}+L_{105}, L_{107}=L_{2}^{1}, L_{108}=L_{3}^{1}, L_{109}=L_{108}^{2}-L_{107}^{2}, \\
& L_{110}=\frac{-L_{108}\left(h_{1}+h_{2}\right)}{\sqrt{\left(\sigma^{2}+M^{2}\right)}}+\frac{2}{\left(\sigma^{2}+M^{2}\right)} L_{107}, \quad L_{111}=\sqrt{\left(\sigma^{2}+M^{2}\right)} L_{108}\left(L_{59}+L_{70}\right)-L_{107}\left(L_{95}+L_{106}\right), \\
& c_{3}=\frac{\frac{1}{\left(\sigma^{2}+M^{2}\right)} \frac{d p_{0}}{d x}\left(h_{2}+h_{1}\right)-\left(L_{59}+L_{70}\right)-c_{4} L_{108}}{L_{107}}, \quad c_{4}=\frac{-\left(\frac{d p_{0}}{d x} L_{10}+L_{111}\right)}{L_{109}}, \\
& L_{112}=\cosh 3 \sqrt{\left(\sigma^{2}+M^{2}\right)} h_{1}-\cosh 3 \sqrt{\left(\sigma^{2}+M^{2}\right)} h_{2}, \\
& L_{113}=\sinh 3 \sqrt{\left(\sigma^{2}+M^{2}\right)} h_{1}-\sinh 3 \sqrt{\left(\sigma^{2}+M^{2}\right)} h_{2}, \\
& L_{114}=\cosh 2 \sqrt{\left(\sigma^{2}+M^{2}\right)} h_{1}-\cosh 2 \sqrt{\left(\sigma^{2}+M^{2}\right)} h_{2}, \\
& L_{115}=h_{1} \cosh 2 \sqrt{\left(\sigma^{2}+M^{2}\right)} h_{1}-h_{2} \cosh 2 \sqrt{\left(\sigma^{2}+M^{2}\right)} h_{2}, \\
& L_{116}=\sinh 2 \sqrt{\left(\sigma^{2}+M^{2}\right)} h_{1}-\sinh 2 \sqrt{\left(\sigma^{2}+M^{2}\right)} h_{2}, \\
& L_{117}=h_{1} \sinh 2 \sqrt{\left(\sigma^{2}+M^{2}\right)} h_{1}-h_{2} \sinh 2 \sqrt{\left(\sigma^{2}+M^{2}\right)} h_{2}, \\
& L_{118}=\frac{L_{5} L_{112}+L_{6} L_{113}}{4}, \quad L_{119}=\frac{L_{114}\left(L_{71}+L_{72}-L_{35}\right)}{2 \sqrt{\left(\sigma^{2}+M^{2}\right)}}, \\
& L_{120}=\frac{\left(L_{73}+L_{79}\right)}{\sqrt{\left(\sigma^{2}+M^{2}\right)}}+\frac{2\left(L_{75}+L_{81}\right)}{\left(\sigma^{2}+M^{2}\right)}+\frac{24\left(L_{77}+L_{83}\right)}{\left(\sigma^{2}+M^{2}\right)^{5 / 2}} .
\end{aligned}
$$




$$
\begin{aligned}
& L_{121}=\frac{\left(L_{74}+L_{80}\right)}{\left(\sigma^{2}+M^{2}\right)}+\frac{6\left(L_{76}+L_{82}\right)}{\left(\sigma^{2}+M^{2}\right)^{2}}+\frac{120\left(L_{78}+L_{84}\right)}{\left(\sigma^{2}+M^{2}\right)^{3}}, \quad L_{122}=\frac{2\left(L_{75}+L_{81}\right)}{\left(\sigma^{2}+M^{2}\right)}+\frac{24\left(L_{77}+L_{83}\right)}{\left(\sigma^{2}+M^{2}\right)^{2}} \\
& L_{123}=\frac{\left(L_{74}+L_{80}\right)}{\sqrt{\left(\sigma^{2}+M^{2}\right)}}+\frac{\sigma\left(L_{76}+L_{82}\right)}{\left(\sigma^{2}+M^{2}\right)^{3 / 2}}+\frac{120\left(L_{78}+L_{84}\right)}{\left(\sigma^{2}+M^{2}\right)^{5 / 2}}, \quad L_{124}=\frac{\left(L_{75}+L_{87}\right)}{\sqrt{\left(\sigma^{2}+M^{2}\right)}}+\frac{12\left(L_{77}+L_{83}\right)}{\left(\sigma^{2}+M^{2}\right)^{3 / 2}}, \\
& L_{125}=\frac{3\left(L_{76}+L_{82}\right)}{\left(\sigma^{2}+M^{2}\right)}+\frac{60\left(L_{78}+L_{84}\right)}{\left(\sigma^{2}+M^{2}\right)^{2}}, \quad L_{126}=L_{31}+L_{3}\left(\frac{\left(L_{76}+L_{82}\right)}{\sqrt{\left(\sigma^{2}+M^{2}\right)}}+\frac{20 L_{84}}{\left(\sigma^{2}+M^{2}\right)^{3 / 2}}\right)-4 L_{2} \frac{\left(L_{77}+L_{83}\right)}{\left(\sigma^{2}+M^{2}\right)}, \\
& L_{127}=\frac{L_{3}\left(L_{77}+L_{83}\right)}{\sqrt{\left(\sigma^{2}+M^{2}\right)}}-\frac{5 L_{2}\left(L_{78}+L_{84}\right)}{\left(\sigma^{2}+M^{2}\right)}, \quad L_{128}=L_{118}+L_{119}+L_{3} L_{120}-L_{2} L_{121}, \\
& L_{129}=\left(h_{1}-h_{2}\right)\left(L_{32-} L_{2} L_{122}+L_{3} L_{123}\right)+\left(h_{1}^{2}-h_{2}^{2}\right)\left(L_{3} L_{124}-L_{2} L_{125}-L_{15}\right) \text {, } \\
& L_{130}=\left(h_{1}^{3}-h_{2}^{3}\right) L_{126}+\left(h_{1}^{4}-h_{2}^{4}\right) L_{127}+\frac{\left(h_{1}^{5}-h_{2}^{5}\right)}{\sqrt{\left(\sigma^{2}+M^{2}\right)}} L_{3}, \\
& L_{131}=\frac{\left(h_{1}+h_{2}\right) L_{2} L_{109}+\left(\sigma^{2}+M^{2}\right) L_{110} L_{108}-\left(\sigma^{2}+M^{2}\right) L_{3} L_{107}-\left(h_{1}-h_{2}\right) L_{107} L_{109}}{\left(\sigma^{2}+M^{2}\right) L_{107} L_{109}}, \\
& L_{132}=L_{128}+L_{129}+L_{130}+\left(\frac{L_{117}\left(L_{3} L_{107}-L_{108}\right)-L_{109} L_{2}\left(L_{59}+L_{70}\right)}{L_{107} L_{109}}\right), \\
& a_{1}=\frac{c_{1}^{2}+c_{2}^{2}}{8\left(\sigma^{2}+M^{2}\right)}, a_{2}=\frac{c_{1} c_{2}}{4\left(\sigma^{2}+M^{2}\right)}, a_{3}=\frac{c_{2}^{2}-c_{1}^{2}}{4}, D_{1}=a_{1}\left(\cosh 2 \sqrt{\sigma^{2}+M^{2}} h_{1}-\cosh 2 \sqrt{\sigma^{2}+M^{2}} h_{2}\right), \\
& D_{2}=a_{2}\left(\sinh 2 \sqrt{\sigma^{2}+M^{2}} h_{1}-\sinh 2 \sqrt{\sigma^{2}+M^{2}} h_{2}\right), D_{3}=a_{3}\left(h_{1}^{2}-h_{2}^{2}\right), \quad D_{4}=a_{1} \cosh 2 \sqrt{\sigma^{2}+M^{2}} h_{1}, \\
& D_{5}=a_{2} \sinh 2 \sqrt{\sigma^{2}+M^{2}} h_{1}, \quad D_{6}=a_{3} h_{1}^{2}, A_{1}=\frac{1}{h_{1}-h_{2}}\left[\left(\sigma^{2}+M^{2}\right)^{2} \operatorname{Br}\left(D_{1}+D_{2}+D_{3}\right)-1\right], \\
& A_{2}=\left(\sigma^{2}+M^{2}\right)^{2} \operatorname{Br}\left(D_{4}+D_{5}+D_{6}\right)-A_{1} h_{1}, \quad A_{3}=\frac{2 E c \operatorname{Pr}\left(\sigma^{2}+M^{2}\right) k_{56}-k_{57}}{h_{1}-h_{2}}, \\
& A_{4}=2 \mathrm{Ec} \operatorname{Pr}\left(\sigma^{2}+M^{2}\right) k_{56}-A_{3} h_{1}, \quad s_{1}=c_{3}\left(\sigma^{2}+M^{2}\right)+L_{73} \sqrt{\sigma^{2}+M^{2}}+L_{80},
\end{aligned}
$$




$$
\begin{aligned}
& s_{2}=L_{74} \sqrt{\sigma^{2}+M^{2}}+2 L_{81}, s_{3}=L_{75} \sqrt{\sigma^{2}+M^{2}}+3 L_{82}, s_{4}=L_{76} \sqrt{\sigma^{2}+M^{2}}+4 L_{83} \text {, } \\
& s_{5}=L_{77} \sqrt{\sigma^{2}+M^{2}}+5 L_{84}, s_{6}=c_{4}\left(\sigma^{2}+M^{2}\right)+L_{74}+L_{79} \sqrt{\sigma^{2}+M^{2}}, \\
& s_{7}=2 L_{75}+L_{80} \sqrt{\sigma^{2}+M^{2}}, s_{8}=3 L_{76}+L_{81} \sqrt{\sigma^{2}+M^{2}}, s_{9}=4 L_{77}+L_{82} \sqrt{\sigma^{2}+M^{2}} \text {, } \\
& s_{10}=5 L_{78}+L_{83} \sqrt{\sigma^{2}+M^{2}}, s_{11}=2\left(L_{71}+L_{35}\right) \sqrt{\sigma^{2}+M^{2}}+4 L_{33}\left(\sigma^{2}+M^{2}\right), \\
& s_{12}=2\left(L_{72}+L_{33}\right) \sqrt{\sigma^{2}+M^{2}}+4 L_{35}\left(\sigma^{2}+M^{2}\right) \\
& k_{1}=\frac{s_{1}}{8\left(\sigma^{2}+M^{2}\right)}+\frac{4 s_{3}+15 s_{5}}{16\left(\sqrt{\sigma^{2}+M^{2}}\right)^{3}}+\frac{s_{7}}{8\left(\sqrt{\sigma^{2}+M^{2}}\right)^{3}}+\frac{18 s_{9}}{64\left(\sqrt{\sigma^{2}+M^{2}}\right)^{5}}+\frac{9 L_{5}}{32\left(\sqrt{\sigma^{2}+M^{2}}\right)}+\frac{60 L_{84}}{32\left(\sqrt{\sigma^{2}+M^{2}}\right)^{3}}, \\
& k_{2}=\frac{s_{6}}{8\left(\sigma^{2}+M^{2}\right)}+\frac{s_{2}}{8\left(\sqrt{\sigma^{2}+M^{2}}\right)^{3}}+\frac{3 s_{8}}{16\left(\sigma^{2}+M^{2}\right)^{2}}+\frac{3 s_{10}}{4\left(\sigma^{2}+M^{2}\right)^{3}}-\frac{9 L_{6}}{32\left(\sqrt{\sigma^{2}+M^{2}}\right)}+\frac{60 L_{78}}{32\left(\sigma^{2}+M^{2}\right)^{3}}, \\
& k_{3}=\frac{s_{2}}{8\left(\sigma^{2}+M^{2}\right)}+\frac{3 s_{4}}{4\left(\sigma^{2}+M^{2}\right)^{2}}+\frac{s_{8}}{4\left(\sqrt{\sigma^{2}+M^{2}}\right)^{3}}+\frac{6 s_{10}+16 L_{78}}{4\left(\sqrt{\sigma^{2}+M^{2}}\right)^{5}}, \\
& k_{4}=\frac{s_{3}}{4\left(\sqrt{\sigma^{2}+M^{2}}\right)^{3}}+\frac{s_{7}}{8\left(\sigma^{2}+M^{2}\right)}+\frac{9 s_{9}}{16\left(\sigma^{2}+M^{2}\right)^{2}}+\frac{15 s_{5}+30 L_{84}}{8\left(\sqrt{\sigma^{2}+M^{2}}\right)^{5}}, \\
& k_{5}=\frac{s_{3}}{8\left(\sigma^{2}+M^{2}\right)}+\frac{24 s_{5}+6 s_{9}}{\left(\sqrt{\sigma^{2}+M^{2}}\right)^{3}}+\frac{60 L_{84}}{16\left(\sigma^{2}+M^{2}\right)^{2}}, \\
& k_{6}=\frac{6 s_{4}}{16\left(\sqrt{\sigma^{2}+M^{2}}\right)^{3}}+\frac{s_{8}}{8\left(\sigma^{2}+M^{2}\right)}+\frac{18 s_{10}+75 L_{78}}{16\left(\sigma^{2}+M^{2}\right)^{2}}, \quad k_{7}=\frac{s_{4}}{8\left(\sigma^{2}+M\right)}+\frac{s_{10}+5 L_{84}}{2\left(\sqrt{\sigma^{2}+M^{2}}\right)^{3}}, \\
& k_{8}=\frac{s_{9}}{8\left(\sigma^{2}+M\right)}+\frac{4 s_{5}+15 L_{84}}{8\left(\sqrt{\sigma^{2}+M^{2}}\right)^{3}}, \quad k_{9}=\frac{2 s_{5}+5 L_{84}}{8\left(\sigma^{2}+M\right)}, \quad k_{10}=\frac{s_{10}+5 L_{78}}{8\left(\sigma^{2}+M\right)}, \quad k_{11}=\frac{c_{1} L_{78}+c_{2} L_{84}}{8\left(\sqrt{\sigma^{2}+M^{2}}\right)}, \\
& k_{12}=\frac{c_{2} L_{78}+c_{1} L_{84}}{8\left(\sqrt{\sigma^{2}+M^{2}}\right)}, \quad k_{13}=\frac{s_{11}}{18\left(\sigma^{2}+M\right)}+\frac{4 L_{35}}{27\left(\sqrt{\sigma^{2}+M^{2}}\right)}, \quad k_{14}=\frac{s_{12}}{18\left(\sigma^{2}+M\right)}+\frac{4 L_{33}}{27\left(\sqrt{\sigma^{2}+M^{2}}\right)},
\end{aligned}
$$




$$
\begin{aligned}
& k_{15}=\frac{s_{11}}{2\left(\sigma^{2}+M\right)}+\frac{4 L_{35}}{\left(\sqrt{\sigma^{2}+M^{2}}\right)}, \quad k_{16}=\frac{s_{12}}{2\left(\sigma^{2}+M\right)}+\frac{4 L_{33}+2 L_{15}}{\left(\sqrt{\sigma^{2}+M^{2}}\right)}, \quad k_{17}=c_{1} L_{33}-c_{2}\left(2 L_{35}-6 L_{31}\right), \\
& k_{18}=\frac{9\left(c_{1} L_{5}+c_{2} L_{6}\right)=}{128 \sqrt{\sigma^{2}+M^{2}}}, k_{19}=\frac{9\left(c_{2} L_{5}+c_{1} L_{6}\right)}{128 \sqrt{\sigma^{2}+M^{2}}}, \quad k_{20}=\frac{\left(c_{1} s_{1}-c_{2} s_{6}\right)}{4}, k_{21}=\frac{\left(c_{1} s_{2}-c_{2} s_{7}\right)}{4}, k_{22}=\frac{7\left(c_{1} s_{3}-c_{2} s_{8}\right)}{24}, \\
& k_{23}=\frac{15\left(s_{4}-s_{9}\right)}{40}, k_{24}=\frac{\left(29 s_{5}-47 s_{10}\right)}{60}, k_{25}=\sqrt{\sigma^{2}+M^{2}}\left(c_{1} L_{78}-c_{2} L_{84}\right), \quad k_{26}=c_{1} L_{33}-c_{2} k_{17} \\
& k_{27}=c_{2} L_{33}-c_{1} k_{17}, \quad k_{28}=c_{1} k_{15}-c_{2} k_{16}, \quad k_{29}=c_{2} k_{15}-c_{1} k_{16}, \quad k_{30}=c_{1} k_{1}-c_{2} k_{2}, k_{31}=c_{1} k_{3}+c_{2} k_{4} \text {, } \\
& k_{32}=c_{1} k_{5}+c_{2} k_{6}, \quad k_{33}=c_{1} k_{7}+c_{2} k_{8}, \quad k_{34}=c_{1} k_{9}+c_{2} k_{10}, \quad k_{35}=c_{1} k_{2}+c_{2} k_{1}, \quad k_{36}=c_{1} k_{4}+c_{2} k_{3} \text {, } \\
& k_{37}=c_{1} k_{6}+c_{2} k_{5}, \quad k_{38}=c_{1} k_{8}+c_{2} k_{7}, \quad k_{39}=c_{1} k_{10}+c_{2} k_{9}, \quad k_{40}=c_{1} k_{13}+c_{2} k_{14}, \quad k_{41}=\frac{2}{9}\left(c_{1} L_{33}+c_{2} L_{35}\right), \\
& k_{42}=c_{1} k_{14}+c_{2} k_{13}, \quad k_{43}=\frac{2}{9}\left(c_{1} L_{35}+c_{2} L_{33}\right), \\
& k_{44}=\cosh \sqrt{\sigma^{2}+M^{2}} h_{l}\left(k_{28}+h_{1} k_{26}\right)+\sinh \sqrt{\sigma^{2}+M^{2}} h_{1}\left(k_{29}+h_{1} k_{27}\right), \\
& k_{45}=\cosh 2 \sqrt{\sigma^{2}+M^{2}} h_{1}\left(k_{30}+h_{1} k_{31}+k_{32} h_{1}^{2}+k_{33} h_{1}^{3}+k_{34} h_{1}^{4}+k_{11} h_{1}^{5}\right), \\
& k_{46}=\sinh 2 \sqrt{\sigma^{2}+M^{2}} h_{1}\left(k_{35}+h_{1} k_{36}+k_{37} h_{1}^{2}+k_{38} h_{1}^{3}+k_{39} h_{1}^{4}+k_{12} h_{1}^{5}\right), \\
& k_{47}=\cosh 3 \sqrt{\sigma^{2}+M^{2}} h_{1}\left(k_{41}+k_{42} h_{1}\right)+\sinh 3 \sqrt{\sigma^{2}+M^{2}} h_{1}\left(k_{42}+k_{43} h_{1}\right), \\
& \left.k_{48}=\cosh 4 \sqrt{\sigma^{2}+M^{2}} h_{1} k_{18}+\sinh 4 \sqrt{\sigma^{2}+M^{2}} h_{1} k_{19}\right), \\
& k_{49}=k_{20} h_{1}{ }^{2}+k_{21} h_{1}^{3}+k_{22} h_{1}^{4}+k_{23} h_{1}^{5}+k_{24} h_{1}^{6}+k_{25} h_{1}^{7},
\end{aligned}
$$




$$
\begin{aligned}
& k_{50}=\cosh \sqrt{\sigma^{2}+M^{2}} h_{2}\left(k_{28}+h_{2} k_{26}\right)+\sinh \sqrt{\sigma^{2}+M^{2}} h_{2}\left(k_{29}+h_{2} k_{27}\right), \\
& k_{51}=\cosh 2 \sqrt{\sigma^{2}+M^{2}} h_{2}\left(k_{30}+h_{1} k_{32}+k_{32} h_{2}^{2}+k_{33} h_{2}^{3}+k_{34} h_{2}^{4}+k_{11} h_{2}^{5}\right), \\
& k_{52}=\sinh 2 \sqrt{\sigma^{2}+M^{2}} h_{2}\left(k_{35}+h_{2} k_{36}+k_{37} h_{2}{ }^{2}+k_{38} h_{2}^{3}+k_{39} h_{2}^{4}+k_{12} h_{2}^{5}\right), \\
& k_{53}=\cosh 3 \sqrt{\sigma^{2}+M^{2}} h_{2}\left(k_{41}+k_{42} h_{2}\right)+\sinh 3 \sqrt{\sigma^{2}+M^{2}} h_{2}\left(k_{42}+k_{43} h_{2}\right), \\
& \left.k_{54}=\cosh 4 \sqrt{\sigma^{2}+M^{2}} h_{2} k_{18}+\sinh 4 \sqrt{\sigma^{2}+M^{2}} h_{2} k_{19}\right), \\
& k_{55}=k_{20} h_{2}^{2}+k_{21} h_{2}^{3}+k_{22} h_{2}^{4}+k_{23} h_{2}^{5}+k_{24} h_{2}^{6}+k_{25} h_{2}^{7}, \\
& k_{56}=k_{44}+k_{45}+k_{46}+k_{47}+k_{48}+k_{49}, k_{57}=k_{50}+k_{51}+k_{52}+k_{53}+k_{54}+k_{55} .
\end{aligned}
$$

\section{References}

[1] Misra J.C. and Pandey S.K. (1999): Peristaltic transport of a non-Newtonian fluid with a peripheral layer. - Int. J. Engng. Sci., vol.37, pp.1841-58.

[2] Usha S. and Rao A.R. (2000): Effect of curvature and inertia on the peristaltic transport in a two fluid system. - Int. J. Engng. Sci., vol.38, pp.1355-75.

[3] Mishra M. and Rao A.R. (2004): Peristaltic transport of a power law fluid in a porous tube. - J. Non-Newtonian Fluid Mech., vol.121, pp.163-74.

[4] Hayat T., Noreen S., Ali N. and Abbasbanday S. (2012): Peristaltic motion of Phan-Thien-Tanner fluid in a planar channel. - Numerical Methods for Partial Differential Equations, vol.28, pp.737-748.

[5] Noreen Sher Akbar and Nadeem S. (2012): Peristaltic flow of a Phan-Thien-Tanner nanofluid in a diverging tube. Heat Transfer - Asian Research, vol.41, pp.10-12.

[6] Vajravelu K., Sreenadh S., Lakshminarayana P., Sucharitha G. and Rashidi M.M. (2015): Peristaltic flow of PhanThien-Tanner fluid in an asymmetric channel with porous medium. - Journal of Applied Fluid Mechanics, Accepted.

[7] Vajravelu K., Radhakrishnamacharya G. and Radhakrishnamurty V. (2007): Peristaltic flow and heat transfer in a vertical porous annulus, with long wave length approximation. - Int. J. Non-Linear Mech., vol.42, pp.754-759.

[8] Srinivas S. and Gayathri R. (2009): Peristaltic transport of a Newtonian fluid in a vertical asymmetric channel with heat transfer and porous medium. - Appl. Math. Comput., vol.215, pp.185-196.

[9] Vajravelu K., Sreenadh S. and Lakshminarayana P. (2011): The influence of heat transfer on peristaltic transport of a Jeffrey fluid in a vertical porous stratum. - Commun. Non-Linear Sci. Numer. Simulat., vol.16, pp.3107-3125.

[10] Sreenadh S., Lakshminarayana P. and Sucharitha G. (2011): Peristaltic flow of micropolar fluid in an asymmetric channel with permeable walls. - Int. J. of Appl. Math. Mech., vol.7, pp.18-37.

[11] Vajravelu K., Sreenadh S., Sucharitha G. and Lakshminarayana P. (2014): Peristaltic transport of a conducting Jeffrey fluid in an inclined asymmetric channel. - Int. J. Biomath., vol.7, 1450064 (25 pages).

[12] Mekheimer Kh.S. (2004): Peristaltic flow of blood under the effect of magnetic field in a non uniform channels. Appl. Math. Comput., vol.153, pp.763-777. 
[13] Hayat T. and Ali N. (2008): Peristaltic motion of a Jeffrey fluid under the effect of a magnetic field in a tube. Commun. Nonlinear Sci. Num. Simulat., vol.13, pp.1343-1352.

[14] Kothandapani M. and Srinivas S. (2008): Peristaltic transport of a Jeffrey fluid under the effect of magnetic field in an asymmetric channel. - Int. J. Nonlinear Mech., vol.43, pp.915-924.

[15] Noreen S., Hayat T. and Alsaedi A. (2011): Study of slip and induced magnetic field effects on peristaltic flow of pseudoplastic fluid. - Int. J. Phys Sci., vol.6, pp.8018-8026.

[16] Hakeem Abd EI. and Nabby Abd EI. (2009): Creeping Flow of Phan-Thien-Tanner fluids in a peristaltic tube with an infinite long wavelength. - ASME J. Applied Mechanics, vol.76, 064504 (6 pages).

[17] Eldabe N.T.M, El-Sayed M.F., Ghaly A.Y. and Sayed H.M. (2007): Peristically induced transport of an MHD biviscosity fluid in a non-uniform tube. - Physica A, vol.383, pp.253-66.

Received: August 15, 2015

Revised: September 10,2015 